\title{
Flexibility in Ceteris Paribus Reasoning
}

\author{
Jeremy Seligman \\ Department of Philosophy, \\ The University of Auckland \\ Private Bag 920i9, Auckland il 42 \\ New Zealand \\ j.seligman@auckland.ac.nz \\ Patrick Girard* \\ Department of Philosophy, \\ The University of Auckland \\ Private Bag 920i9, Auckland il 42 \\ New Zealand \\ p.girard@auckland.ac.nz \\ Received by Greg Restall \\ Published December 2I, 2OII
}

http://www.philosophy · unimelb.edu.au/aj1/2011

(C) 20II Jeremy Seligman and Patrick Girard

\begin{abstract}
Ceteris Paribus clauses in reasoning are used to allow for defeaters of norms, rules or laws, such as in von Wright [5]'s example "I prefer my raincoat over my umbrella, everything else being equal”.

In $[3,4]$, a logical analysis is offered in which sets of formulas $\Gamma$, embedded in modal operators, provide necessary and sufficient conditions for things to be equal in ceteris paribus clauses. For most laws, the set of things allowed to vary is small, often finite, and so $\Gamma$ is typically infinite. Yet the axiomatisation they provide is restricted to the special and atypical case in which $\Gamma$ is finite.

We address this problem by being more flexible about ceteris paribus conditions, in two ways. The first is to offer an alternative, slightly more general semantics, in which the set of formulas only give necessary but not (necessarily) sufficient conditions. This permits a simple axiomatisation.
\end{abstract}

*The authors are grateful for the extensive hospitality and facilities of South West University, Chongqing, and Tsinghua University, Beijing, at which this paper was conceived and completed. In particular, they acknowledge support of the National Social Science Foundation of China (o9Czx033), the Foundation for Humanities and Social Sciences by the Ministry of Education of China (08JC72040002) and the Fundamental Research Funds of Southwest University (swu $90955^{12}$ ). They also thank the anonymous referee, whose fast, comprehensive and insightful report has improved the paper greatly. 
The second is to consider those sets of formulas which are sufficiently flexible to allow the construction of a satisfying model in which the stronger necessary-andsufficient interpretation of $[3,4]$ is maintained.

The language $\mathrm{L}_{c p}$ of ceteris paribus logic, proposed in [3, 4], is intended to represent propositions that are true only under the assumption that certain conditions remain constant. It is a generalisation of earlier work by von Wright [5] concerning ceteris paribus preferences. Suppose, for example, that I prefer white wine to red. It does not follow that I prefer white wine while being beaten with an iron bar to red wine while sitting on my deck reading a book. My preference only holds 'other things being equal'. $\mathrm{L}_{\mathfrak{c p}}$ is a propositional modal logic with an operator $\langle\Gamma\rangle$ for each set of formulas $\Gamma$, which is intended to represent the conditions that are kept constant.

[4] provides a simple axiomatisation of the set of those valid formulas of $\mathrm{L}_{c p}$ that contain only modalities $\langle\Gamma\rangle$ for finite $\Gamma$. We'll call such $\langle\Gamma\rangle$ finite modalities. The full language, however, has modalities of arbitrary size. This paper presents an axiomatisation of part of the full logic, the set of what we call 'flexible' validities, and provides an alternative semantics $L_{c p}$ for which all validities are flexible, and so completely axiomatised

Before explaining these concepts any further, it is worth countering a few objections. One might think that any investigation of the infinite modalities has purely technical interest, at best. We are used to philosophical applications of logic, especially to the behaviour of rational agents, that typically involves reasoning about finite situations using finite languages. There are two aspects to this objection that are particularly revealing in the present case. Firstly, the restriction to finite situations makes a good deal of sense for the most part. Consideration of ceteris paribus statements is an exception.

When I say that I prefer white wine to red, we expect a logical analysis to be restricted to the concepts expressed by parts of the statement: white, red, wine, preference, and me. But the 'ceteris paribus' clause adds an indefinite realm of things that have to be held constant in the evaluation of the statement. In some cases, it may be possible to restrict this to another finite set, but we think it prudent to explore the general case first. Secondly, the restriction to finite languages (languages with finitely long formulas) is well-motivated by considerations of human comprehensibility. An infinite formula is unreadable. It would be better, perhaps, to extend the language with finite terms that refer to the infinite sets of ceteris paribus conditions. For example, we could add a new modal operator Prop〉 in which 'Prop' is a symbol denoting the set of all propositional variables. This operator would express a very strong ceterisparibus condition that all atomic propositions are held constant without needing to list them all inside the diamond. But the suggestion is quickly followed by a

\footnotetext{
'The idea that repeated uses of the words 'flexible' and 'flexibly' is intended to convey is that ceteris paribus statements have a inherent flexibility that resists a precise characterisation by a rigid set of conditions that are held constant.
} 
very natural question: which of the infinite modalities can be dealt with in this way? Our answer involves consideration of infinite sets of formulas and infinite formulas and a careful study of the relationship between the two.

There is a third role for the infinite in our approach. We are interested in characterising which ceteris paribus statements follow from a potentially infinite amount of information. In more familiar terms, we want a strong completeness result. Weak completeness for $\mathrm{L}_{\mathfrak{c p}}$, namely a finite characterisation of the set of $\mathrm{L}_{c p}$ validities, remains an open problem, and we hope that the work presented here will assist in its solution. But there is reason for wanting more. To the extent that we are right in thinking that ceteris paribus statements involve implicit reference to a potentially infinite range of things to be held constant, we must also attempt to understand how the truth of a ceteris paribus statement depends (or does not depend) on that infinite range.

In outline, the rest of the paper will go as follows. First, in Section I, we introduce the language of $\mathrm{L}_{c p}$ and its semantics. We distinguish between two classes of models, the class of flexible ceteris paribus models, over which we state a strong completeness theorem, and the more restricted class of ceteris paribus models, which coincides with the logic studied in previous work. The logic of ceteris paribus models is not compact and so axiomatising its more tractable fragments becomes the main theme of the rest of the paper.

In a long Section 2 , we study our problem in the setting of a slightly more abstract language $\mathrm{L}(\mathcal{C})$ in which the sets of ceteris paribus conditions inside diamonds are replaced by single symbols called 'parameters', thus returning us to the world of finitely long formulas ${ }^{2}$ Parametric Ceteris Paribus Logic, as we call it, has a simple axiomatisation, which is given in Section 2.I It turns out, however, that some models of $\mathrm{L}(\mathrm{C})$ are such that the ceteris paribus conditions interpreting a given parameter $\mathrm{c}$ cannot be described by any set of formulas in the language. That is to say, there is no set of formulas $\Gamma$ such that $\langle c\rangle$ and $\langle\Gamma\rangle$ have the same interpretation in the model.

In Section 2.2, we give some preliminary results about the class of 'language models': those for which every parameter represents ceteris paribus conditions that can be described using sets of formulas. A finer-grained investigation of language models proceeds in Section 2.3. There we define a 'description' to be a function $\delta$ associating each parameter $c$ with a set $\delta(c)$ of formulas intended to represent the ceteris paribus conditions of c. A $\delta$-model is one in which this intention is realised. In Section 2.4 we define the $\delta$-flexible validities using some axioms and rules which are sound over $\delta$-models. It is not a complete axiomatisation of the set of $\mathrm{L}(\mathrm{C})$ formulas over $\delta$-models for reasons discussed above in the case of $\mathrm{L}_{\mathfrak{c p}}$.

We continue with our unorthodox strategy, identifying sufficient conditions for a set of formulas to be ' $\delta$-flexible' and proving the main result, that

\footnotetext{
${ }^{2}$ Although, 'finite' here should be taken with a pinch of salt. In the general case, we need a proper class of parameters.
} 
any $\delta$-flexible set of formulas that is $\delta$-flexibly consistent has a model. This is done in Section 2.5. With all of this work done in the more abstract setting of $\mathrm{L}(\mathcal{C})$, the next task is to apply it back to the original language of $\mathrm{L}_{\mathrm{cp}}$ and return to the examples listed above. That's Section 3 .

\section{Ceteris Paribus LOG IC}

The language $L_{c p}$ is a language of propositional modal logic with a set Prop of propositional variables and modal operators $\langle\Gamma\rangle$ for each set $\Gamma$ of formulas. A model $M$ for $L_{c p}$ is a tuple $\langle W, R, \approx, V\rangle$ where $W$ is a set of states, $R$ a binary accessibility relation on $\mathrm{W}$ and $\mathrm{V}$ a valuation function from Prop to subsets of W.

The remaining component $\approx$ is a family of equivalence relations $\approx_{\Gamma}$ on $W$, with $\Gamma$ a set of formulas. The formulas in $\Gamma$ contain the information that is to be kept equal. There are two ways to understand this latter statement. In the first instance, we may require that the formulas in $\Gamma$ be kept equal between two states $u$ and $v$ whenever $u \approx_{\Gamma} v$. In the second instance, we may require that the set $\Gamma$ precisely defines when two states are equivalent, so that $u \approx_{\Gamma} v$ if and only if the formulas in $\Gamma$ are kept equal. We call the first kind flexible ceteris paribus equivalence, the second ceteris paribus equivalence.

More precisely, a flexible ceterisparibus equivalence for a set of formulas $\Gamma$ is an equivalence relation on $W$ with the following constraint:

If $u \approx_{\Gamma} v$, then for each $\varphi \in \Gamma, M, u \models \varphi$ iff $M, v \models \varphi$.

A model with this property will be called a flexible ceteris paribus model. A ceterisparibus equivalence is fully defined by sets of formulas $\Gamma$, with the following constraint:

$$
\mathrm{u} \approx_{\Gamma} v \quad \text { iff } \quad \text { for each } \varphi \in \Gamma, M, u \models \varphi \text { iff } M, v \models \varphi
$$

A model with this property will be called ceteris paribus model. In this case, $u \approx_{\Gamma} v$ holds just in case states $u$ and $v$ satisfy exactly the same formulas in $\Gamma$. In either case, the equivalence relation is used to interpret the modal operators:

$$
M, u \models\langle\Gamma\rangle \varphi \quad \text { iff } \quad R u v \text { and } u \approx_{\Gamma} v \text { and } M, v \models \varphi \text { for some } v \in W
$$

These definitions are not obviously well-formed, so we will need to be a little more precise, constructing $\mathrm{L}_{c p}$ and $\models$ in stages. For each ordinal $\alpha$, we define $L_{\alpha}$ to be

$$
\varphi::=p(p \in \operatorname{Prop})|\neg \varphi|(\varphi \vee \varphi) \mid\langle\Gamma\rangle \varphi\left(\Gamma \subseteq \mathrm{L}_{\beta} \text { for some } \beta<\alpha\right) \sqrt{3}
$$

The satisfaction relation $\models_{\alpha}$ is defined as follows:

\footnotetext{
${ }^{3}$ So, in particular, $\mathrm{L}_{0}$ is the language of propositional logic with variables in Prop, $\mathrm{L}_{1}$ has also modalities of the form $\langle\Gamma\rangle$ for each set $\Gamma \subseteq \mathrm{L}_{0}, \mathrm{~L}_{\omega}$ has modalities of the form $\langle\Gamma\rangle$ for each set $\Gamma \subseteq \mathrm{L}_{\mathrm{n}}$ for some $\mathrm{n}$, and so on. Notice that if $\alpha \leqslant \gamma$ then $\mathrm{L}_{\alpha} \subseteq \mathrm{L}_{\gamma}$.
} 


$$
\begin{array}{lll}
M, u \models_{\alpha} p & \text { iff } & u \in V(p) \\
M, u \models_{\alpha} \neg \varphi & \text { iff } & M, u \models_{\alpha} \varphi \\
M, u \models_{\alpha}(\varphi \vee \psi) & \text { iff } & M, u \models_{\alpha} \varphi \text { or } M, u \models_{\alpha} \psi \\
M, u \models_{\alpha}\langle\Gamma\rangle \varphi & \text { iff } & R u v \text { and } u \approx_{\Gamma} v \text { and } M, v \models_{\alpha} \varphi \text { for some } v \in W
\end{array}
$$

Note that each formula of $\Gamma$ is in $L_{\beta}$ for some $\beta<\alpha$, so $\approx_{\Gamma}$ is defined at an earlier stage. The ordinal sequences $\mathrm{L}_{\alpha}$ and $\models_{\alpha}$ are both monotonic increasing and so we can define $\mathrm{L}_{c p}$ and $\models$ to be their respective unions. (Both are proper classes.)

Standard abbreviations are used. In particular, the universal modality $[\Gamma]$ is defined to be $\neg\langle\Gamma\rangle \neg$.

Henceforth, we will abuse notation slightly by using $L_{c p}$ for the logic of this language interpreted over ceteris paribus models, and $\mathrm{L}_{\mathrm{cpf}}$ for the logic over flexible ceteris paribus models. Both can be regarded as extensions of basic modal logic by defining $\square=[\emptyset]$ and $\diamond=\langle\emptyset\rangle$. Indeed, the machinery of modal model theory can be and has been applied to $\mathrm{L}_{c p}$. [4] gives a number of results about $\mathrm{L}_{\mathrm{cp}}$ : the logic is invariant under bisimulation; it doesn't have the finite model property; it can be translated into basic modal logic with infinite disjunctions and it is independent from propositional dynamic logic (PDL).

One important difference between $\mathrm{L}_{c p}$ and its flexible counterpart $\mathrm{L}_{c p f}$ is that the latter is strongly axiomatisable, whereas the former is not. Consider a simple case where $\Gamma=$ Prop, the set of all propositional variables. The following set of formulas $\Delta$ is satisfiable in flexible ceteris paribus models, but not in ceteris paribus models:

$$
\Delta=\operatorname{Prop},\{\square \mathrm{p} \mid \mathrm{p} \in \operatorname{Prop}\}, \diamond \mathrm{q}, \neg\langle\operatorname{Prop}\rangle \mathrm{q}
$$

To see this, consider the following flexible model, in which we suppose that $u \neq$ Prop $v$ :

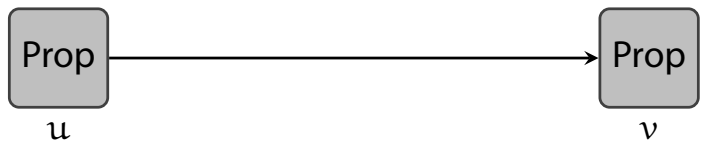

In this model, $u \models$ Prop and $u \models \square p$ for each $p \in$ Prop and also $u \models \diamond q$. But $u \not \forall\langle$ Prop $\rangle$ q, because $u \not \not_{\text {Prop }} v$ even though both $u$ and $v$ satisfy all of Prop.

In ceterisparibus models, however, we can show that $\Delta$ is unsatisfiable. Consider a state $u$ at which every propositional variable $p$ and every formula $\square p$ is satisfied. If $\nabla q$ also holds at $u$ then there is an accessible state $v$ at which $q$ holds. Moreover, every other propositional variable $p$ must also hold at $v$, because $\square p$ holds at $u$ and Ruv. Thus $u \approx_{\text {Prop }} v$. But then $\langle\operatorname{Prop}\rangle q$ holds at $u$ and so $\neg\langle$ Prop $\rangle$ q cannot.

But every finite subset $\Delta_{0}$ of $\Delta$ is satisfiable because at least one propositional variable (call it $r$ ) is missing. (It occurs in $\langle$ Prop $\rangle$ but not as a formula 
in $\Delta_{0}$.) We can satisfy $\Delta_{0}$ at $u$ in the model shown below, with $R=\{\langle u, v\rangle\}$, $V(r)=\{v\}$ and $V(p)=W$ if $p \neq r$.

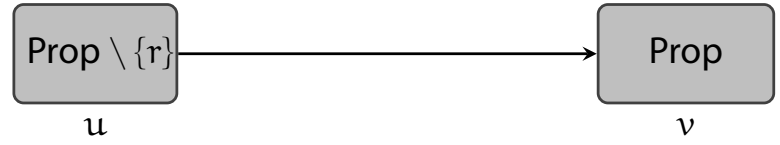

Any system of axioms and finitary rules defines a compact consequence relation and so there can be no such system for $\mathrm{L}_{\mathrm{cp}}$.

This paper is mainly concerned with strong axiomatisations of fragments of $\mathrm{L}_{c p}$, which we will make precise below. For $\mathrm{L}_{\mathfrak{c p f}}$, we can readily obtain a strong axiomatisation, and thus compactness, based on the following two axioms:

$$
\begin{array}{ll}
\text { (CP-Preservation) } & \vdash \varphi \rightarrow[\Gamma] \varphi \text { and }\langle\Gamma\rangle \varphi \rightarrow \varphi \text { for all } \varphi \in \Gamma . \\
\text { (CP-Inclusion) } & \vdash\langle\Gamma\rangle \varphi \rightarrow\left\langle\Gamma^{\prime}\right\rangle \varphi \text { for each } \Gamma^{\prime} \subseteq \Gamma
\end{array}
$$

THEOREM I Flexible ceterisparibus logic, $\mathrm{L}_{\mathrm{cpf}}$, is strongly axiomatised by $\mathrm{CP}-\mathrm{Inclusion}$ and $\mathrm{CP}-$ Preservation together with the axioms and rules of normal modal logic.

We will come back to a fuller discussion of these two axioms in Section 2.I and will prove Theorem $\mathrm{I}$ as a consequence of Lemma 5 below. We now focus on the more difficult task of axiomatising fragments of $\mathrm{L}_{\mathrm{cp}}$.

One fragment that has already been shown to be strongly complete in [4] is the finitary fragment of $\mathrm{L}_{c p}$, in which all operators $\langle\Gamma\rangle$ are built from a finite set $\Gamma$. The finitary fragment is axiomatised by the axioms and rules of basic modal logic together with

$$
\langle\psi, \Gamma\rangle \varphi \quad \leftrightarrow \quad(\psi \wedge\langle\Gamma\rangle(\psi \wedge \varphi)) \vee(\neg \psi \wedge\langle\Gamma\rangle(\neg \psi \wedge \varphi))
$$

This one axiom scheme is sufficient to provide a recursive translation of the finitary fragment into basic modal logic, and so completeness is obvious. We can eliminate the ceteris paribus operators in one go using infinitary logic. If $\Gamma=\left\{\psi_{i}\right\}_{i \in \mathrm{I}}$ then we have

$$
\langle\Gamma\rangle \varphi \quad \leftrightarrow \quad \bigvee_{\alpha \in \Gamma^{*}}(\alpha \wedge \diamond(\alpha \wedge \varphi))
$$

where $\Gamma^{*}=\left\{\bigwedge_{i \in \mathrm{I}} \pm \psi_{i} \mid \pm \psi_{i}=\psi_{i}\right.$ or $\left.\neg \psi_{i}\right\}$ is the set of all possible conjunctions of formulas in $\Gamma$ (or their negations). In the finitary case, the righthand side is a formula of basic modal logic; in the general case, it is a formula of infinitary basic modal logic.

This finitary restriction only offers a partial solution to the problem of axiomatising $L_{c p}$, as ceteris paribus clauses typically involve an infinite amount of information. Instead, we offer another strategy.

We will provide (finitely characterisable) sufficient conditions for a set of formulas not to be counterexample to compactness. A set of formulas satisfying these conditions will be called a 'flexible set'. Our main theorem (Theorem 
I8) then has the following form: any flexible set of formulas that is flexibly consistent is satisfiable. By 'flexibly consistent' we mean that the set does not contain formulas $\varphi_{1}, \ldots, \varphi_{n}$ such that $\neg\left(\varphi_{1} \wedge \cdots \wedge \varphi_{n}\right)$ is a flexible validity, for which we have a finite characterisation.

This approach is somewhat unorthodox but we hope it might be useful in other branches of infinitary logic. The interest lies in the fact that the definition of 'flexible set of formulas' and 'flexible validity' differ in a way that indicates that we are discovering some real structure in the logical morass of this infinitary language, and one that sheds some light on the nature of ceteris paribus statements. So before developing the theory, we will list some examples of flexible sets of formulas. We ask the reader to keep these examples in mind when reading the subsequent definitions and theorems. We will come back to them in Section 3.I.

Let $\Gamma$ be a set of formulas of $L_{c p}$ and let $\operatorname{Prop}(\Gamma)$ be the set of propositional variables that occur in $\Gamma$ outside modal operators 4 The following are sufficient conditions for $\Gamma$ to be flexible.

I. $\Gamma$ contains only $\langle\Delta\rangle$ for finite $\Delta$. This is the logic axiomatised in [4]. There is no restriction on the formulas occurring in $\Delta$. They may be complex formulas, also containing modal operators, and there can be non-trivial logical relations between the formulas inside the operators and those outside. But, importantly, $\Delta$ is restricted to being a finite set. As discussed above, this is a significant limitation because applications (e.g., to preference logic) typically require that the 'other things' held constant constitute an infinite set. For example, in formalising the statement ' $\varphi$ is preferred to $\psi$ ', von Wright requires that all propositional variables other than those occurring in $\varphi$ and $\psi$ remain constant.

2. $\Gamma$ contains only one modality $\langle\Delta\rangle$ in which $\Delta=\operatorname{Prop} \backslash \operatorname{Prop}(\Gamma)$. In this case, we will show that finitary inferences are sufficient for determining the satisfiability of $\Gamma$. It is an important example, because it gives one direct way of making sense of 'ceteris paribus', in which the 'other things' are all propositional variables not mentioned in the argument.

3. $\Gamma$ contains many modalities $\left\langle\Delta_{i}\right\rangle$, with $\Delta_{0} \subset \Delta_{1} \subset \cdots \subseteq \operatorname{Prop} \backslash \operatorname{Prop}(\Gamma)$. Slightly generalising the previous example, we can consider a chain of ceterisparibus modalities of increasing strength. Again, finitary inferences are sufficient to characterise the logic of these modalities so long as there is no interaction between the variables inside the modal operators and those outside.

4. $\Gamma$ contains only the modalities $\langle\operatorname{Prop}\rangle$ and $\langle\emptyset\rangle$ and $\operatorname{Prop}(\Gamma)$ is finite. If there are only a finite number of propositional variables outside the scope

${ }^{4}$ All of this will be defined carefully in the following sections. Hopefully, it is clear enough even at this point for the reader to get a general idea. 
of a modal operator then inferences about the maximal and minimal $c e-$ teris paribus operators are finitely characterisable. The restriction on the number of propositional variables in $\operatorname{Prop}(\Gamma)$ is essential as we will see shortly.

5. $\Gamma$ contains modalities $\langle\Delta\rangle$ in which $\Delta$ is any set of propositional variables, only a finite number of which are in $\operatorname{Prop}(\Gamma)$.

6. $\Gamma$ contains a finite set of modalities $\left\langle\Delta_{1}\right\rangle, \ldots,\left\langle\Delta_{n}\right\rangle$ in which each $\Delta_{i}$ contains a cofinite set of propositional variables only finitely many of which are in $\operatorname{Prop}(\Gamma)$. We will see in Section 3.I that this is a generalisation of von Wright's ceteris paribus logic.

7. $\Gamma=\{\varphi\}$ with $\varphi$ containing only modalities with cofinite sets of propositional variables. This is a special case of the previous example, and an important one for applications. Note in particular that in this case the modal operators may contain propositional variables that also occur outside modal operators.

\section{PARAMETRIC ceteris paribus LOGIC}

Our strategy for studying $L_{c p}$ is to move to a more tractable logic in which the sets of formulas inside the modal operators are replaced by something more abstract, and then to examine how $\mathrm{L}_{\mathfrak{c p}}$ arises as a special case. So, instead of sets of formulas inside our diamonds, we will use parameters, drawn from a preordered class $\mathcal{C}=\langle\mathrm{C}, \leqslant\rangle$. As before, the elements of $\mathrm{C}$ represent conditions that must be held constant across a ceteris paribus modal transition, and $\mathrm{c} \leqslant \mathrm{d}$ means that parameter $c$ represents a less restrictive condition than $d$. If $c \leqslant d$ for all $\mathrm{d} \in \mathrm{D} \subseteq \mathcal{C}$ then we say that $\mathrm{c}$ is a least element of $\mathrm{D}$. Least elements will play a significant role in some of our later results.

Define $\mathrm{L}(\mathrm{C})$ to be the language of propositional modal logic with propositional variables Prop and modal operators $\langle c\rangle$ for each $c \in C$. The original ceteris paribus logic arises as the special case in which $\mathrm{C}=\wp\left(\mathrm{L}_{\mathrm{cp}}\right), \leqslant=\subseteq$ and $\mathrm{L}(\mathrm{C})=\mathrm{L}_{\mathrm{cp}}$.

A model for $\mathrm{L}(\mathrm{C})$ is a tuple $M=\langle\mathrm{W}, \mathrm{R}, \approx, \mathrm{V}\rangle$ where $\mathrm{W}$ is a set of states, $R$ a binary accessibility relation on $W$ and $V$ a valuation function from Prop to subsets of $W$. For each $c \in C, \approx_{c}$ is an equivalence relation on $W$ such that

(Ordering) if $c \leqslant d$ then for all $u, v \in W$, if $u \approx_{d} v$ then $u \approx_{c} v$.

In other words, if $\mathrm{c}$ is less restrictive than $\mathrm{d}$ then whenever two states are equivalent with respect to $d$, they are also equivalent with respect to c. Satisfaction is defined as usual but with

$$
M, u \models\langle c\rangle \varphi \text { iff there is a } v \text { such that } R u v \text { and } u \approx_{c} v \text {, and } M, v \models \varphi .
$$


The crucial difference from $\mathrm{L}_{\mathfrak{c} p}$ is that the meaning of 'other things being equal' is specified in the model rather than in the language, using the equivalence relations $\approx_{\mathrm{c}}$.

As usual, the satisfaction of formulas depends only on the vocabulary that they contain, including the parameters. Given a formula $\varphi$ of $\mathrm{L}(\mathcal{C})$, we say that models $M=\langle W, R, \approx, V\rangle$ and $M^{\prime}=\left\langle W, R, \approx^{\prime}, V^{\prime}\right\rangle$ agree on $\varphi$ if:

I. $V(p)=V^{\prime}(p)$ for each propositional variable $p$ in $\varphi$

2. $\mathrm{R} \cap \approx_{\mathrm{c}}=\mathrm{R} \cap \approx_{\mathrm{c}}^{\prime}$ for each parameter $\mathrm{c}$ in $\varphi$

LEMma 2 (Agreement) If $M$ and $M^{\prime}$ agree on $\varphi$, then $M, u \models \varphi$ iff $M^{\prime}, \mathfrak{u} \models \varphi$.

Proof: By induction on the structure of $\varphi$.

Notice that a model of $\mathrm{L}(\mathrm{C})$ can also be regarded as a standard model for multi-modal propositional logic in which the operator $\langle\mathrm{c}\rangle$ is represented by the accessibility relation $R_{c}=R \cap \approx_{c}$, so that

$$
M, u \models\langle c\rangle \varphi \quad \text { iff } \quad R_{c} u v \text { and } M, v \models \varphi \text { for some } v \in W
$$

A structure of the form $\left\langle W,\left\{R_{c}\right\}_{c} \in C, V\right\rangle$ will be called a normal model. It will be convenient to work with normal models so that standard results from modal logic can be applied. Note, however, that not all normal models are models for $\mathrm{L}(\mathrm{C})$.

LEMMA 3 Let $\mathrm{M}$ be a model of $\mathrm{L}(\mathcal{C})$ and let $\mathrm{M}^{\mathrm{N}}$ be a normal model for $\mathrm{M}$. Then $\mathrm{M}, \mathrm{u} \models \varphi$ iff $\mathrm{M}^{\mathrm{N}}, \mathrm{u} \models \varphi$ for any $\varphi$.

Proof: By induction on the structure of $\varphi$.

\section{I AXIOMATISATION}

The only new logical principle that we get for parametric ceterisparibus logic is one that keeps track of the ordering of parameters:

$$
\text { (Inclusion) } \vdash\langle\mathrm{d}\rangle \varphi \rightarrow\langle\mathrm{c}\rangle \varphi \quad \text { for each } \mathrm{c} \leqslant \mathrm{d}
$$

THEOREM 4 The logic of $\mathrm{L}(\mathrm{C})$ is axiomatised by Inclusion together with the normal rules and axioms for basic modal logic.5

As usual, the theorem is a consequence of a model existence lemma, which is a little stronger than required here but whose strength will be needed later. LEMMA 5 If $\Gamma$ is a set of formulas of $\mathrm{L}(\mathcal{C})$ that is consistent with respect to Inclusion and the rules and axioms of basic modal logic, then there is a model $M=\langle W, R, \approx, V\rangle$ and a state $w \in W$ such that $M, w \models \Gamma$. Moreover, $R$ has the structure of a tree with root $w$, and the property that if $\mathrm{u} \approx_{\mathrm{c}} v$ and $\mathrm{u} \neq v$ then either Ruv or $\mathrm{R} v \mathrm{u}$.

${ }^{5}$ We take the axioms and rules of basic modal logic to be all propositional tautologies, $(\mathrm{K})$ $[c](p \rightarrow q) \rightarrow([c] p \rightarrow[c] q),(M P)$ from $\varphi$ and $(\varphi \rightarrow \psi)$ infer $\psi$, and (Gen) from $\varphi$ infer $[c] \varphi$. 
Proof: Suppose $\Gamma$ is consistent and $C$ is a set ${ }^{6}$ Take the canonical structure $M=\left\langle W,\left\{R_{c}\right\}_{c} \in C, V\right\rangle$ with $W$ the set of maximal consistent subsets of $L(C)$ and relations $R_{c}$ defined in the usual way: $R_{c} u v$ iff for all $\varphi$ in $v,\langle c\rangle \varphi$ is in $u$. This is a normal model which may not be an $L(\mathcal{C})$ model. In a standard way, we can prove that there is a maximal consistent extension $\Gamma^{+}$of $\Gamma$ such that $\Gamma$ is satisfied at $\Gamma^{+}$(which is a member of $W$ ) when the modalities are interpreted as normal modal operators. Let $R$ be the union of all the $R_{c}$ and define $N=\langle W, R, V\rangle$. Now unravel $N$ with respect to $R$ from the state $\Gamma^{+}$to get the structure $N^{\prime}=\left\langle W^{\prime}, R^{\prime}, V^{\prime}\right\rangle$, which is a tree whose root we will call $w, 7$ For each $u$ in $W^{\prime}$, let $u^{\prime}$ be its source in $M$ and define $R_{c}^{\prime} u v$ iff $R_{c} u^{\prime} v^{\prime}$. In particular, $w^{\prime}=\Gamma^{+}$. The normal model $M^{\prime}=\left\langle W^{\prime},\left\{R_{c}^{\prime}\right\}_{c} \in C, V^{\prime}\right\rangle$ has the following properties:

I. $\mathcal{M}^{\prime}, \mathfrak{u} \models \varphi$ iff $M, \mathfrak{u}^{\prime} \models \varphi$ for every $\varphi \in \mathrm{L}(\mathcal{C})$.

2. $R^{\prime}=U_{c} R_{c}^{\prime}$ is irreflexive, asymmetric and intransitive (if Rxy and Ryz then not $R x z$ ).

3. $R_{d}^{\prime} \subseteq R_{c}^{\prime}$ iff $c \leqslant d$

Property $\mathrm{I}$ follows from the fact that $M^{\prime}, u$ is bisimilar to $M, u^{\prime}$, by the unravelling construction, which also ensures 2 Property 3 is due to Inclusion in the right-to-left direction. For the left-to-right direction, note that if $\mathrm{c} \nless \mathrm{d}$ then $(\langle d\rangle p \wedge \neg\langle c\rangle p)$ is consistent and so can be extended to a point of the canonical model that is $R_{d}$-related to a point where $p$ holds but which is not $R_{c}$ related to any such point.

Now we build an $L(\mathcal{C})$ model $M^{\prime \prime}=\left\langle W^{\prime}, R^{\prime},\left\{\approx_{c}\right\}_{c} \in C, V^{\prime}\right\rangle$ by taking $\approx_{c}$ to be the smallest equivalence relation containing $R_{c}^{\prime}$. That this is an $L(\mathcal{C})$ model follows from 3 , above. We only need to verify that $M^{\prime \prime}, w$ is equivalent to $M^{\prime}, w$ and hence to $M, w^{\prime}$, so that $\Gamma$ is satisfied in $M^{\prime \prime}$ at $w$. That is, we must show that for every $u \in W^{\prime}$ and $\varphi \in \mathrm{L}(\mathcal{C})$.

$$
M^{\prime \prime}, u \models \varphi \text { iff } M^{\prime}, u \models \varphi
$$

This is done by induction on the structure of $\varphi$. The only non-trivial case is when $\varphi$ has the form $\langle c\rangle \psi$. Given the $\mathrm{L}(\mathcal{C})$ interpretation of $\langle c\rangle$, it suffices to show that $R^{\prime \prime} \cap \approx_{c}=R_{c}^{\prime}$.

That $R_{c}^{\prime} \subseteq R^{\prime \prime} \cap \approx_{c}$ follows directly from the definitions of $R^{\prime \prime}$ and $\approx_{c}$. For the converse, suppose for contradiction that $R^{\prime \prime} u v$ and $u \approx_{c} v$ but not $R_{c}^{\prime} u v$. Then $\approx_{\mathrm{c}}^{\prime}=\approx_{\mathrm{c}} \backslash\langle u, v\rangle$ includes $R_{\mathrm{c}}^{\prime}$. By construction, $\approx_{\mathrm{c}}^{\prime}$ is not reflexive only if

${ }^{6}$ If $C$ is a proper class, we carry out the construction using $L\left(\mathcal{C}_{\Gamma}\right)$ instead of $L(\mathcal{C})$, where $\mathcal{C}_{\Gamma}$ is the set of parameters occurring in $\Gamma$. When we have an $L\left(\mathcal{C}_{\Gamma}\right)$ model that satisfies $\Gamma$, we can extend it to a model for $\mathrm{L}(\mathcal{C})$ by defining $\approx_{\mathrm{c}}$ for $\mathrm{c} \in \mathrm{C} \backslash \mathrm{C}_{\Gamma}$ more-or-less arbitrarily. We need only ensure that the $\leqslant$ ordering is preserved, i.e. that $\approx_{\mathrm{d}} \subseteq \approx_{\mathrm{c}}$ if $\mathrm{c} \leqslant \mathrm{d}$. This can be done by setting $\approx_{\mathrm{d}}=\bigcap\left\{\approx_{\mathrm{c}} \mid \mathrm{c} \leqslant \mathrm{d}\right.$ and $\left.\mathrm{c} \in \mathrm{C}_{\Gamma}\right\}$.

${ }^{7}$ Unravelling is described in [2] p. 62-63]. 
$u=v$. But since $R^{\prime \prime}$ is irreflexive and $R^{\prime \prime} u v, u \neq v$ and so $\approx_{c}^{\prime}$ is reflexive. Likewise, $\approx_{c}^{\prime}$ is symmetric and transitive because $R^{\prime \prime}$ is asymmetric and intransitive. But then $\approx_{c}^{\prime}$ is an equivalence relation containing $R_{c}^{\prime}$ which is strictly smaller than $\approx_{\mathfrak{c}}$, contradicting the definition of $\approx_{\mathfrak{c}}$.

Finally, if $u \approx_{c} v$ and $u \neq v$ then $R^{\prime \prime} u v$ or $R^{\prime \prime} v u$. Otherwise, $\approx_{c}$ would not be the smallest equivalence relation extending $R_{c}^{\prime}$.

As promised, we return to the proof of Theorem Ir repeated here:

$$
\begin{array}{ll}
\text { (CP-Preservation) } & \vdash \varphi \rightarrow[\Gamma] \varphi \text { and }\langle\Gamma\rangle \varphi \rightarrow \varphi \text { for all } \varphi \in \Gamma \text {. } \\
\text { (CP-Inclusion) } & \vdash\langle\Gamma\rangle \varphi \rightarrow\left\langle\Gamma^{\prime}\right\rangle \varphi \text { for each } \Gamma^{\prime} \subseteq \Gamma
\end{array}
$$

THEOREM I Flexible ceterisparibus logic, $\mathrm{L}_{\mathrm{cpf}}$, is strongly axiomatised by CP-Inclusion and CP-Preservation together with the axioms and rules of normal modal logic.

Proof: Suppose that $\Delta$ is consistent with respect to the above-mentioned rules and axioms. Recall that with $\mathcal{C}$ taken to be sets of formulas of $L_{c p}$ ordered by $\subseteq$, we can identify the language of $\mathrm{L}_{\mathfrak{c p}}$ with $\mathrm{L}(\mathcal{C})$. Applying Lemma $5, \Delta$ is satisfied by a state $w$ of a model $M=\langle W, R, \approx, V\rangle$. Moreover,

$$
\text { (*) If } u \approx_{\Gamma} v \text { then either Ruv or Rvu. }
$$

What remains to be shown is that $M$ is a flexible ceteris paribus model, i.e. that $u \approx_{\Gamma} v$ implies that for all $\varphi \in \Gamma, M, u \models \varphi$ iff $M, v \models \varphi$. So assume that $u \approx_{\Gamma} v$ and that $M, u \models \varphi$. By $\left({ }^{*}\right)$, either Ruv or Rvu. In the first case, using the CP-Preservation axiom $\mathrm{M}, \mathrm{u} \models \varphi \rightarrow[\Gamma] \varphi$, we get that $\mathrm{M}, v \models \varphi$. In the second case, we get instead that $M, v \models\langle\Gamma\rangle \varphi$, but the CP-Preservation axiom $\langle\Gamma\rangle \varphi \rightarrow \varphi$ then implies that $M, u \models \varphi$. Since the argument is entirely symmetrical between $u$ and $v$, we get that for all $\varphi \in \Gamma, M, u \models \varphi$ iff $M, v \models \varphi$, as desired.

\subsection{LANGUAGE MODELS}

An obvious question to ask of an $\mathrm{L}(\mathcal{C})$ model is whether equivalence relations $\approx_{c}$ can be described by sets of formulas. If they can, we get one step closer to the language $L_{c p}$. So, say that an $L(C)$ model $M=\langle W, R, \approx, V\rangle$ is a language model if for each $c \in C$ there is a set $\Sigma_{c}$ of formulas such that

$$
u \approx_{c} v \text { iff for all } \varphi \text { in } \Sigma_{\mathfrak{c}}, M, u \models \varphi \text { iff } M, v \models \varphi .
$$

Restricting to the class of language models for $\mathrm{L}(\mathcal{C})$ gives a strictly stronger logic.

THEOREM 6 Not every satisfiable set offormulas of $\mathrm{L}(\mathrm{C})$ bas a language model.

Proof: Suppose $\mathcal{C}$ has only conditions $c$ and $d$ with $c \leqslant d$ but not $d \leqslant c$. Consider the two-point model $M_{2}$ with Ruv and Rvu and $u \approx_{c} v$ but not $u \approx_{d} v$, and an empty propositional valuation. 


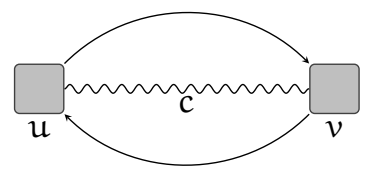

Note that $u$ and $v$ are indistinguishable and so satisfy the same formulas. Now let $T_{2}$ be the theory of $M_{2}, u$ in $L(\mathcal{C})$. Suppose, for contradiction, that $T_{2}$ is satisfied at point $w$ of language model $M$. Then $\langle c\rangle \top$ is in $T_{2}$ so $M, w \models\langle c\rangle \top$ and so there is a $w^{\prime}$ such that Rww $w^{\prime}$ and $w \approx_{\mathrm{c}} w^{\prime}$. The formula $\langle\mathrm{d}\rangle \perp$ is also in $T_{2}$, so $w \nsim_{\mathrm{d}} w^{\prime}$. Thus there is a formula $\varphi$ in $\Sigma_{\mathrm{d}}$ such that $M, w \models \varphi$ and $M, w^{\prime} \not \models \varphi$. But then $\varphi$ is in $T_{2}$, so $M_{2}, u \models \varphi$ and $M_{2}, v \models \varphi$. Then $M_{2}, u \models$ $[c] \varphi$, and so $[\mathrm{c}] \varphi$ is also in $T_{2}$, and so $M, w \models[c] \varphi$, which is incompatible with $\mathrm{R} w w^{\prime}, w \approx_{c} w^{\prime}$ and $M, w^{\prime} \not \neq \varphi$.

An example of a rule of inference that is sound for language models but not arbitrary models can be constructed as follows. Let $P(c, d)$ be the class of formulas $\langle\mathrm{d}\rangle(\psi \wedge\langle\mathrm{c}\rangle \neg \psi)$ for each $\psi$ such that $\psi \rightarrow[\mathrm{c}] \psi$ is $\mathrm{L}(\mathcal{C})$-invalid $]^{8}$ Then the rule states:

$$
\text { (PN) } P(c, d),\langle d\rangle \varphi \quad\langle c\rangle \varphi
$$

Before proving this, we prove a simple lemma which will be used again below.

LEMMA 7 If $\varphi$ and $\neg \varphi$ are $\mathrm{L}(\mathcal{C})$-invalid for some $\varphi$, then $\langle\mathrm{c}\rangle \varphi \rightarrow[\mathrm{c}] \varphi$ is also $\mathrm{L}(\mathrm{C})$ invalid.

Proof: Suppose $\varphi$ and $\neg \varphi$ are $\mathrm{L}(\mathcal{C})$-invalid for some $\varphi$, then there are two rooted models $M, u$ and $M^{\prime}, v$ such that $M, u \models \varphi$ and $M^{\prime}, v \models \neg \varphi$ for some $u, v$. Now, construct a new model $M^{\prime \prime}$ by taking the disjoint union of $M$ and $M^{\prime}$ with an additional point $w$ with Rwu and Rwv, and $w \approx_{\mathrm{c}} \mathrm{u}, w \approx_{\mathrm{c}} v$ and $u \approx_{c} v$ (as shown on the left, below):

\footnotetext{
${ }^{8}$ One might question whether this is a genuine rule of inference, on the grounds that $P(c, d)$ is semantically defined. In the case that $\mathcal{C}$ is countable, $\mathrm{L}(\mathcal{C})$ is decidable (because it has a complete axiomatisation and obviously has the finite model property, since Inclusion is preserved when taking submodels), and so $\mathrm{P}(\mathrm{c}, \mathrm{d})$ could also be defined in an entirely syntactic manner. When we move to uncountable $\mathcal{C}$, or worse, a $\mathcal{C}$ that is a proper class, this argument does not work; moreover, even if $\mathrm{P}(\mathrm{c}, \mathrm{d})$ were syntactically defined, the fact that it is a proper class stretches the boundaries of what we ordinarily think of as a rule of inference. Fortunately, nothing in what follows depends on this classification. We will not be proposing PN as part of any system of logic.
} 


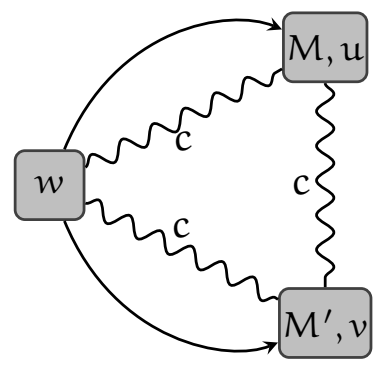

$M^{\prime \prime}$

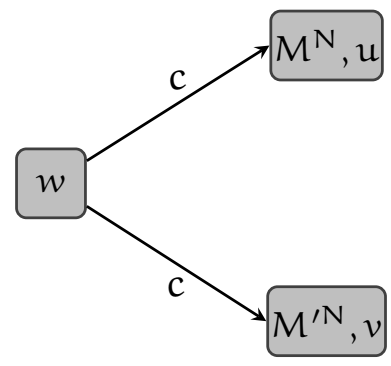

$M^{\prime \prime N}$

By Lemma 3, there is an equivalent normal modal $M^{\prime \prime N}$, with $R_{c} w u$ and $R_{c} w v$ but neither $R_{c} u v$ nor $R_{c} v u$, as shown on the right. Now, the generated model of $M^{\prime \prime N}$ at $u$ is just $M^{N}$ which (by Lemma 3) agrees with $M$ on $\varphi$, and the generated model $M^{\prime \prime N}$ at $v$ is just $M^{\prime N}$ which (by Lemma 3) agrees with $M^{\prime}$ on $\varphi$, so (again by Lemma 3. $M^{\prime \prime}, \mathfrak{u} \models \varphi$ and $M^{\prime \prime}, v \models \neg \varphi$. Therefore, $M^{\prime \prime}, w \models$ $\langle c\rangle \varphi$, but $M^{\prime \prime}, w \not \models[c] \varphi$.

Now for the main theorem of this section:

THEOREM 8 PN is sound for language models but unsound over arbitrary models.

Proof: For a counterexample to PN, notice that if $\psi \rightarrow[c] \psi$ is $L(\mathcal{C})$-invalid, then there is a pointed model $\left\langle P_{\psi}, p_{\psi}\right\rangle$ of $\psi \wedge\langle c\rangle \neg \psi$. So let $M$ be a model consisting of the disjoint union of all the $\mathrm{P}_{\psi}$, together with a point $u$ such that $\operatorname{Rup}_{\psi}$ and $u \approx_{d} p_{\psi}$.

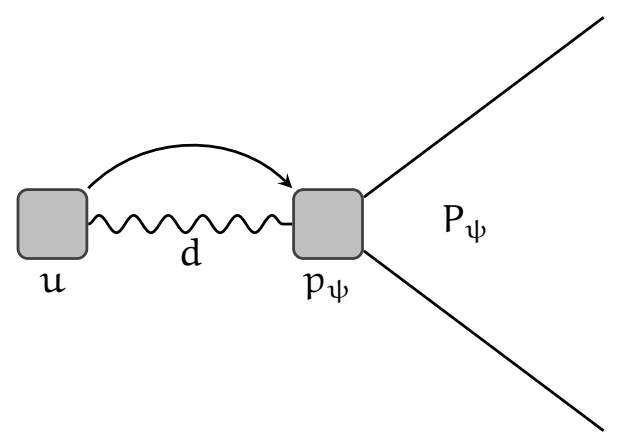

Since the model can be normalised, in a manner similar to the proof of Lemma 7 we can be sure that the truth value of a formula at $p_{\psi}$ in $M$ are the same as its value at $p_{\psi}$ in $P_{\psi}$. Now $u \neq_{c} p_{\psi}$, so the conclusion $\langle c\rangle T$ is not satisfied by $u$. But $u \models\langle d\rangle \top$ and for each $\langle d\rangle(\psi \wedge\langle c\rangle \neg \psi)$ in $P(c, d)$, we have that $p_{\psi} \models \psi \wedge\langle c\rangle \neg \psi$. Since $u \approx_{d} p_{\psi}$, all the premises are satisfied by $u$.

Now, suppose that $M, u$ satisfies the premises $P(c, d)$ and $\langle d\rangle \varphi$, and there is a description $\Sigma_{c}$ of $c$. Then there is a $v$ in $M$ such that $R_{d} u v$ and $M, v \models$ $\varphi$. To conclude that $M, u \models\langle c\rangle \varphi$, we will show that $u \approx_{c} v$. Suppose for contradiction that $u \nsim_{c} v$. Then there is a $\psi \in \Sigma_{c}$ such that $M, u \models \psi$ and 
$M, v \models \neg \psi$ or $M, u \models \neg \psi$ and $M, v \models \psi$. In either case, $\psi$ and $\neg \psi$ are $\mathrm{L}(\mathcal{C})-$ invalid. But this implies that $\langle\mathrm{c}\rangle \psi \rightarrow[\mathrm{c}] \psi$ is also $\mathrm{L}(\mathcal{C})$-invalid, from Lemma 7

So we cannot have that both $\psi \rightarrow[\mathrm{c}] \psi$ and $\langle\mathrm{c}\rangle \psi \rightarrow \psi$ are $\mathrm{L}(\mathcal{C})$-valid, and so either $\langle d\rangle(\psi \wedge\langle c\rangle \neg \psi)$ is in $P(c, d)$ or $\langle d\rangle(\neg \psi \wedge\langle c\rangle \neg \neg \psi)$ is in $P(c, d)$. Then there is a point $w$ in $M$ such that Ruw and which satisfies either $\psi \wedge\langle c\rangle \neg \psi$ or $\neg \psi \wedge\langle c\rangle \neg \neg \psi$. In either case, there is a s such that $w \approx_{c} s$ but $\psi$ is not preserved between $w$ and $s$, contradicting our choice of $\psi \in \Sigma_{c}$.

\subsection{DESCRIPTIONS}

To study language models further, we need to refer to ways of associating parameters with descriptions of them. So we'll say that a description $\delta$ for $\mathcal{C}$ is a function that maps each $c \in \mathcal{C}$ to a set $\delta(c)$ of $L(\mathcal{C})$ formulas such that $\delta(\mathrm{c}) \subseteq \delta(\mathrm{d})$ if $\mathrm{c} \leqslant \mathrm{d}$.

A model $M$ is described by $\delta$ if it satisfies the following condition:

$$
u \approx_{c} v \text { iff for all } \varphi \in \delta(\mathrm{c}), M, u \models \varphi \text { iff } M, v \models \varphi
$$

A model $M$ is a $\delta$-model if $\delta$ is a description of $M$. Clearly, every $\delta$-model is a language model, so we already have an example of a model that is not a $\delta$ model and a rule of inference that is sound for all $\delta$-models. Nonetheless, in some circumstances, we can change a model into a $\delta$-model. Given a model $M=\langle W, R, \approx, V\rangle$, let $[\delta] M$ be the model $\left\langle W, R, \approx_{[\delta]}, V\right\rangle$ where

$$
u \approx_{[\delta] \mathrm{c}} v \text { iff for each } \varphi \in \delta(\mathrm{c}), M, u \models \varphi \text { iff } M, v \models \varphi
$$

This satisfies the Ordering condition, and so $[\delta] M$ is a model. In general, we cannot be sure that it is a $\delta$-model 9 But if, for example, a condition $c$ is such that $\delta(\mathrm{c})$ consists of formulas without any conditions at all, Agreement Lemma 2 ensures that the $\delta$-model property for $\mathrm{c}$, at least, is satisfied in $[\delta] \mathrm{M}$. We say that such parameters have 'depth' zero. Parameters whose description contains only parameters of depth zero have depth one, and so on. That is, we define a sequence $\mathcal{C}_{0}, \mathcal{C}_{1}, \ldots$ of sets of parameters, where

$\mathcal{C}_{\alpha}=$ the set of those $c \in \mathcal{C}$ such that for every $\varphi \in \delta(\mathrm{c})$ and $\mathrm{d} \in \mathcal{C}$, if $\mathrm{d}$ occurs in $\varphi$ then $\mathrm{d} \in \mathcal{C}_{\beta}$ for some $\beta<\alpha$.

The depth of $\mathrm{c}$ is the smallest ordinal $\alpha$ such that $\mathrm{c} \in \mathcal{C}_{\alpha}$. For any formula $\varphi$, the depth of $\varphi$ is the smallest ordinal $\alpha$ such that every parameter occurring in $\varphi$ has depth $<\alpha$. Note that having no depth is different from having depth 0 . We say that $\delta$ is well-founded iff every parameter has a depth. IO

\footnotetext{
${ }^{9}$ For example, suppose $\mathcal{C}=\{\mathrm{c}, \mathrm{d}\}, \delta(\mathrm{c})=\emptyset, \delta(\mathrm{d})=\{\langle\mathrm{c}\rangle \top\}$, and $M$ is the two-state model with $W=\{u, v\}, R=\{\langle u, v\rangle\}$, and $\approx_{c}=\approx_{d}=$ the identity relation on $W$. Then neither $u$ nor $v$ satisfy $\langle c\rangle \top$ in $M$, so $u \approx_{[\delta] d} v$. But also $u \approx_{[\delta] c} v$ so $[\delta] M, u \models\langle c\rangle \top$ and $[\delta] M, v \not \models\langle c\rangle \top$.

${ }^{10}$ This is equivalent to the well-foundedness (in the standard sense) of the relation that holds between $d$ and $c$ iff $d$ occurs in some formula of $\delta(c)$.
} 
We can now give a way of constructing $\delta$-models for well-founded $\delta$, which we will use later in the service of our completeness results. The basic idea is to apply $[\delta]$ repeatedly over an ordinal sequence, taking limits judiciously:

$$
\begin{aligned}
& {\left[\delta^{0}\right] M }=M \\
& {\left[\delta^{\alpha+1}\right] M }=[\delta]\left[\delta^{\alpha}\right] M \\
& {\left[\delta^{\lambda}\right] M }=\left\langle W, R, \approx_{\left[\delta^{\lambda}\right]}, V\right\rangle \\
& \text { with } \approx_{\left[\delta^{\lambda}\right] c}= \begin{cases}\approx_{\left[\delta^{\alpha+1}\right] c} & \text { if } c \text { has depth } \alpha<\lambda \\
\approx_{c} & \text { otherwise }\end{cases}
\end{aligned}
$$

Finally, the limit $\delta^{\infty}$ is defined similarly to the limit-ordinal case:

$$
\left[\delta^{\infty}\right] M=\left\langle W, R, \approx_{\left[\delta^{\infty}\right]}, V\right\rangle
$$

where for each parameter $\mathrm{c}$ of depth $\alpha, \approx_{\left[\delta^{\infty}\right] \mathrm{c}}=\approx_{\left[\delta^{\alpha+1}\right] \mathrm{c}}$.

The following technical lemmas show that this is a sensible definition.

LEMma $9 \quad$ (i) $\quad\left[\delta^{\infty}\right] M, w \models \varphi \quad$ iff $\quad\left[\delta^{\alpha}\right] M, w \models \varphi \quad$ for all $\varphi$ of depth $\leqslant \alpha$.

$$
\text { (ii) } \quad \approx_{\left[\delta^{\infty}\right] \mathrm{c}} \quad=\quad \approx_{\left[\delta^{\alpha+1}\right] \mathrm{c}} \quad \text { for all } \mathrm{c} \text { of depth } \leqslant \alpha .
$$

Proof: By induction on $\alpha$.

(i) $\left[\delta^{\infty}\right] M$ and $\left[\delta^{\alpha}\right] M$ have the same valuation, so the result follows from the Agreement Lemma 2 if we can show that for any parameter $\mathrm{d}$ occurring in $\varphi$,

$$
\mathrm{R} \cap \approx_{[\delta \infty] \mathrm{d}}=\mathrm{R} \cap \approx_{\left[\delta^{\alpha}\right] \mathrm{d}}
$$

Let $\beta$ be the depth of $d$. Then $\beta<\alpha$. There are three cases, depending on whether $\alpha$ is 0 , a successor or a limit ordinal. If $\alpha=0$, there is no such parameter $\mathrm{d}$ in $\varphi$, so there is nothing to prove. If $\alpha=\gamma+1$, then $\gamma<\alpha$, so by inductive hypothesis (ii),

$$
\approx_{\left[\delta^{\infty}\right] \mathrm{d}}=\approx_{\left[\delta^{\gamma+1}\right] \mathrm{d}}
$$

But $\gamma+1=\alpha$, so we are done. Finally, if $\alpha$ is a limit ordinal, then

$$
\approx_{\left[\delta^{\alpha}\right] \mathrm{d}}=\approx_{\left[\delta^{\beta+1}\right] \mathrm{d}}=\approx_{\left[\delta^{\infty}\right] \mathrm{d}} .
$$

(ii) Suppose $c$ has depth $\beta \leqslant \alpha$. If $\beta=\alpha$ then we are done, by definition of $\delta^{\infty}$. So assume $\beta<\alpha$. Then the following are equivalent:

$$
\begin{aligned}
& u \approx \delta_{[\delta \infty] c} v \\
& u \approx_{\left[\delta^{\beta+1}\right] c} v \quad \text { by definition of } \delta^{\infty} \text {, } \\
& {\left[\delta^{\beta}\right] M, u \models \psi \text { iff }\left[\delta^{\beta}\right] M, v \models \psi \quad \text { for all } \psi \in \delta(c), \quad \text { since } c \text { has depth } \beta} \\
& {\left[\delta^{\infty}\right] M, u \models \psi \text { iff }\left[\delta^{\infty}\right] M, v \models \psi \quad \text { for all } \psi \in \delta(c) \text {, by inductive hypothe- }} \\
& \text { sis (i), since the depth } \\
& \text { of } \psi \leqslant \beta<\alpha \\
& {\left[\delta^{\alpha}\right] M, u \models \psi \text { iff }\left[\delta^{\alpha}\right] M, v \models \psi \quad \text { for all } \psi \in \delta(c), \quad \text { by part (i), since the }} \\
& \text { depth of } \psi \leqslant \beta<\alpha \\
& u \approx_{\left[\delta^{\alpha+1}\right] \mathrm{c}} v \\
& \text { by definition of } \delta^{\alpha+1}
\end{aligned}
$$


LEMMA IO

(i) $\left[\delta^{\alpha}\right] M, w \models \varphi \quad$ iff $\quad\left[\delta^{\beta}\right] M, w \models \varphi \quad$ for all $\varphi$ of depth $\leqslant \beta<\alpha$.

(ii) $\quad \approx_{\left[\delta^{\alpha}\right] \mathrm{c}} \quad=\quad \approx_{\left[\delta^{\beta+1}\right] \mathrm{c}} \quad$ for all c of depth $\leqslant \beta<\alpha$.

Proof: Part (i) is a direct consequence of Lemma 9(i). For part (ii), since $\beta<\alpha$, the claim follows from definition if $\alpha$ is a limit ordinal. So assume that $\alpha=$ $\sigma+1$. Then, $\beta \leqslant \sigma$, so $\approx_{\left[\delta^{\alpha}\right] \mathrm{c}}=\approx_{\left[\delta^{\sigma+1}\right] \mathrm{c}}=\approx_{\left[\delta^{\infty}\right] \mathrm{c}}$ by Lemma 9(ii). But $\approx_{\left[\delta^{\infty}\right] \mathrm{c}}=\approx_{\left[\delta^{\beta+1}\right] \mathrm{c}}$, by definition of $\delta^{\infty}$.

LEMMA II If $\delta$ is well-founded then $\left[\delta^{\infty}\right] M$ is a $\delta$-model.

Proof: Suppose $\delta$ is well-founded, then $c \in \mathcal{C}_{\alpha}$ for some $\alpha$. The following are equivalent:

$$
\begin{aligned}
& u \approx\left[\delta^{\infty}\right] \mathrm{c} v \\
& u \approx_{\left[\delta^{\alpha+1}\right] \mathrm{c}} v \quad \text { by definition of }\left[\delta^{\infty}\right] . \\
& {\left[\delta^{\alpha}\right] M, u \models \varphi \text { iff }\left[\delta^{\alpha}\right] M, v \models \varphi \quad \text { for all } \varphi \in \delta(c) \text { by definition of }} \\
& \approx_{\left[\delta^{\alpha+1}\right] \mathrm{c}} \text {. } \\
& {\left[\delta^{\infty}\right] M, u \models \varphi \text { iff }\left[\delta^{\infty}\right] M, v \models \varphi \quad \text { for all } \varphi \in \delta(\mathrm{c}), \quad \text { by Lemma } 9 \text { (i), since }} \\
& \text { the depth of } \varphi \leqslant \text { the } \\
& \text { depth of } c \leqslant \alpha
\end{aligned}
$$

\subsection{AXIOMS FOR L(C) WITH DESCRIPTIONS}

The restriction to a particular description $\delta$ suggests a more specific axiom and rule:

$$
\begin{aligned}
& \text { ( } \delta \text {-Preservation) } \vdash \varphi \rightarrow[\mathrm{c}] \varphi \text { and } \vdash\langle\mathrm{c}\rangle \varphi \rightarrow \varphi \text {, for all } \varphi \in \delta(\mathrm{c}) \text {. } \\
& \text { ( } \delta \text {-Expansion) } \quad \text { If } \vdash(\theta \rightarrow \varphi) \text { or } \vdash(\theta \rightarrow \neg \varphi) \text { for each } \varphi \in \delta(d) \backslash \delta(\mathrm{c}) \\
& \text { then } \vdash(\theta \wedge\langle\mathrm{c}\rangle(\theta \wedge \psi)) \rightarrow\langle\mathrm{d}\rangle \psi
\end{aligned}
$$

The axiom $\delta$-Preservation expresses the defining property of formulas in $\delta(c)$ : that they are preserved across $R_{c}$ links in the model. $\delta$-Expansion is a partial converse. Note that it is a possibly infinitary rule rather than an axiom scheme. We will call the resulting modal logic $L(\mathcal{C})_{\delta}$. It is an extension of $\mathrm{L}(\mathcal{C})$ because Inclusion is derivable. In fact, we get the following strengthened version of Inclusion:

$$
\text { ( } \delta \text {-Inclusion) } \vdash\langle\mathrm{c}\rangle \varphi \rightarrow\langle\mathrm{d}\rangle \varphi \text {, when } \delta(\mathrm{d}) \subseteq \delta(\mathrm{c}) \text {. }
$$

LEMMA I2 LCP is sound for $\delta$-models.

\footnotetext{
${ }^{{ }^{1}}$ When $\delta(\mathrm{d}) \subseteq \delta(\mathrm{c}), \delta(\mathrm{d}) \backslash \delta(\mathrm{c})=\emptyset$, so we can pick $\theta$ to be a tautology.
} 
Proof: The validity of $\delta$-Preservation in $\delta$-models is clear, so we only prove $\delta$-Expansion.

Suppose $M$ is a $\delta$-model and either $\theta \rightarrow \varphi$ or $\theta \rightarrow \neg \varphi$ is valid in $M$ for each $\varphi \in \delta(d) \backslash \delta(c)$. Take $u$ arbitrary such that $M, u \models \theta \wedge\langle c\rangle(\theta \wedge \psi)$. Then $M, \mathfrak{u} \models\langle c\rangle(\theta \wedge \psi)$, so there exists a $v$ such that $u \approx_{c} v$ and $M, v \models \theta \wedge \psi$, and so also $M, v \models \theta$. Now for any $\varphi \in \delta(d)$, either $\varphi \in \delta(\mathrm{c})$ and so $M, u \models \varphi$ iff $M, v \models \varphi$, or $\varphi \in \delta(d) \backslash \delta(\mathrm{c})$. But then $M \models \theta \rightarrow \varphi$ or $M \models \theta \rightarrow \neg \varphi$, and since both $M, u \models \theta$ and $M, v \models \theta$, we also get that $M, u \models \varphi$ iff $M, v \models \varphi$. Thus $\mathrm{u} \approx_{\mathrm{d}} v$. But $M, v \models \psi$, so $M, \mathrm{u} \models\langle\mathrm{d}\rangle \psi$.

The rule PN, which we showed to be sound for language models, but not admissible in $\mathrm{L}(\mathcal{C})$, is admissible in $\mathrm{L}(\mathcal{C})_{\delta}$ :

LEMMA I3 Every $\mathrm{L}(\mathrm{C})_{\delta}$ model validates $\mathrm{PN}$.

Proof: As a reminder, $P(c, d)$ is the set of formulas $\langle d\rangle(\psi \wedge\langle c\rangle-\psi)$ for each $\psi$ such that $\psi \rightarrow[c] \psi$ is $L(\mathcal{C})$-invalid, and $\mathrm{PN}$ is the following rule:

$$
\text { (PN) } \quad P(c, d),\langle d\rangle \varphi \quad \Rightarrow \quad\langle c\rangle \varphi
$$

Suppose that $M$ is a $L(\mathcal{C})_{\delta}$ model and that $P(c, d)$ is satisfied at a point $u$ in $M$. We will show that every $\varphi$ in $\delta(c)$ is either an $L(\mathcal{C})$ validity or an $L(\mathcal{C})$ contradiction, and so the premises of $\delta$-Expansion hold with $\theta$ a tautology. Then, the conclusion is equivalent to $(\langle\mathrm{d}\rangle \varphi \rightarrow\langle\mathrm{c}\rangle \varphi)$, and we are done.

So suppose that $\psi$ is in $\delta(c)$. Since $M$ validates $\delta$-Preservation, $(\psi \rightarrow[c] \psi)$ and $(\langle c\rangle \psi \rightarrow \psi)$ are both satisfied everywhere. So the formulas $\langle d\rangle(\psi \wedge\langle c\rangle-\psi)$ and $\langle d\rangle(\neg \psi \wedge\langle c\rangle \neg \neg \psi)$ are false at $u$, and hence not in the set $P(c, d)$. But then, by the definition of $P(c, d)$, both $(\psi \rightarrow[c] \psi)$ and $(\neg \psi \rightarrow[c] \neg \psi)$ (equivalently $(\langle c\rangle \psi \rightarrow \psi))$ must be $L(\mathcal{C})$ valid. That either $\psi$ or $\neg \psi$ is $L(\mathcal{C})$-valid, follows from Lemma 7 .

Notice that if $\delta(d) \backslash \delta(c)$ is a finite set $\varphi_{1}, \ldots, \varphi_{n}$, the rule $\delta$-Expansion is equivalent to adding axioms

$$
(\theta \wedge\langle\mathrm{c}\rangle(\theta \wedge \psi)) \rightarrow\langle\mathrm{d}\rangle \psi
$$

for each formula $\theta$ of the form $\pm \varphi_{1} \wedge \cdots \wedge \pm \varphi_{n}$, where $\pm \varphi_{i}$ is either $\varphi_{i}$ or $\neg \varphi_{i}$. The rule of $\delta$-Expansion is only a partial converse of $\delta$-Preservation and the three principles are not sufficient for a complete axiomatisation of the logic of $\mathrm{L}(\mathrm{C})$ over $\delta$-models. This can be seen from the following counterexample, adapted from the proof of Theorem $6{ }^{\mathrm{I} 2}$

COUNTEREXAMPLE: Let $\mathcal{C}$ consist of two conditions $c$ and $d$ with $c \leqslant d$ and $d \notin c$, as in the proof of Theorem 6 , and let $\delta(c)$ be

\footnotetext{
${ }^{12}$ Strictly speaking, a counterexample is unnecessary. We can be sure of the inadequacy of $\delta$-preservation from compactness considerations, given that $L_{c p}$ is a special case of $L(\mathcal{C})$.
} 
the empty set and $\delta(d)$ the set of all formulas of $L(\mathcal{C})$. The theory $\mathrm{T}_{2}$ constructed in the proof of Theorem 6 contains all formulas of the form $\varphi \rightarrow[x] \varphi$ and $\langle x\rangle \varphi \rightarrow \varphi$, for $x=c, d$ and so is trivially consistent with $\delta$-Preservation. But it has no language models, and so, a fortiori, no $\delta$-models. So we get a counterexample by showing that $T_{2}$ is also consistent with $\delta$-Expansion. Suppose for contradiction that it is not. Then there is a formula $\theta$ such that for every formula $\varphi$ of $\mathrm{L}(\mathcal{C})$, either $\vdash(\theta \rightarrow \varphi)$ or $\vdash(\theta \rightarrow \neg \varphi)$, and $\neg(\theta \wedge\langle\mathrm{c}\rangle(\theta \wedge \psi)) \rightarrow\langle\mathrm{d}\rangle \psi$ is in $\mathrm{T}_{2}$. Then $\theta \in \mathrm{T}_{2}$ and so $\mathrm{M}_{2}, \mathfrak{u} \models \theta$. Now let $q$ be a propositional variable not in $\theta$ and let $M_{2}^{\prime}$ be like $M_{2}$ but with the complementary valuation for q, i.e. $V^{\prime}(q)=W \backslash V(q)$. By Lemma $2, M_{2}^{\prime}, u \models \theta$. But $M_{2}, u \models q$ iff $M_{2}^{\prime}, u \models \neg q$. And so by Lemma I2, $M_{2}$ and $M_{2}^{\prime}$ provide counterexamples to $\vdash(\theta \rightarrow q)$ and $\vdash(\theta \rightarrow \neg \mathrm{q})$, contradicting the choice of $\theta$.

The problem lies not with PN but with the fact that we have no converse of $\delta$-Preservation. We would like to ensure that if $d \notin c$ then there is a formula $\varphi$ that is guaranteed not to be preserved along $\approx_{\mathrm{c}}$ somewhere in the model. Any such converse will be an infinitary rules (when $\delta(c)$ is infinite) and so is beyond the scope of our present approach.

\section{$2.5 \delta$-FLEXIBILITY}

Instead, we invoke our new strategy and define the concept of a ' $\delta$-flexible' set of formulas, with respect to which we then prove a (flexible) version of strong completeness.

First, some preliminary definitions. For any set of formulas $\Gamma$, let $\operatorname{Prop}(\Gamma)$ be the set of propositional variables that occur in $\varphi$ for some formula $\varphi$ in $\Gamma$. Similarly, for any set $C$ of parameters and description $\delta$, let $\operatorname{Prop}(C, \delta)$ be the set of propositional variables that occur in $\delta(c)$ for some $c \in C$.

Say that a set $C$ of parameters is $\delta$-tracking if $c \leqslant d$ whenever $\delta(c) \subseteq \delta(d)$ for all $c, d \in \delta$. This is the converse of the defining condition for descriptions. Our general concept of a description allows for some leeway between the modalities and their description by sets of formulas but to build models for suitable sets of formulas we need the finer control that comes from $\delta$-tracking.

Say that a set $C$ of parameters is $\delta$-irreducible iff for each $c, \delta(c) \subseteq$ Prop and for each non-empty subset $D \subseteq C$, if $\delta(c) \subseteq \operatorname{Prop}(D, \delta)$ then $\delta(c) \subseteq \delta(d)$ for some $d \in D$. For example, with parameters $c_{0}<c_{1}, c_{2}<c_{3}$ and $\delta$, $\delta^{\prime}$ given by

\begin{tabular}{l|l|l} 
& $\delta$ & $\delta^{\prime}$ \\
\hline$c_{0}$ & $\emptyset$ & $\emptyset$ \\
$c_{1}$ & $\{p\}$ & $\{p\}$ \\
$c_{2}$ & $\{\mathbf{q}\}$ & $\{\mathbf{q}\}$ \\
$c_{3}$ & $\{p, q, r\}$ & $\{p, q\}$
\end{tabular}


$\delta$ is irreducible but $\delta^{\prime}$ is not because $\delta^{\prime}\left(c_{3}\right) \subseteq \delta^{\prime}\left(c_{1}\right) \cup \delta^{\prime}\left(c_{2}\right)$ but neither $\delta\left(c_{3}\right) \subseteq \delta\left(c_{1}\right)$ nor $\delta\left(c_{3}\right) \subseteq \delta\left(c_{2}\right)$. The irreducibility condition is the key to flexibility. If $\mathcal{C}$ is $\delta$-irreducible then the inclusion relation between description sets is determined by a single propositional variable, which we call a 'sprinkle' because of its role in the model-building construction to follow. We say that an assignment of a propositional variable $p_{c}$ (the sprinkle) to each non-least element $c \in \mathcal{C}$ is a $\delta$-sprinkling iff $c \leqslant d$ iff $p_{c} \in \delta(d)$ for each $c, d \in \mathcal{C}$ with $c$ a non-least element.

LEMMA I4 If $\mathrm{C}$ is $\delta$-irreducible and $\delta$-tracking then it has a $\delta$-sprinkling.

Proof: Suppose $\mathrm{c} \in \mathcal{C}$ is a non-least element. Then the set $\mathrm{D}_{\mathrm{c}}=\{\mathrm{d} \in \mathcal{C} \mid \delta(\mathrm{c}) \not \subset$ $\delta(d)\}$ is non-empty by $\delta$-tracking, and $\delta(c) \nsubseteq \operatorname{Prop}\left(D_{c}, \delta\right)$, by $\delta$-irreducibility. Hence, $\delta(c) \backslash \operatorname{Prop}\left(D_{c}, \delta\right) \neq \emptyset$, so we can pick a sprinkle $p_{c} \in \delta(c) \backslash \operatorname{Prop}\left(D_{c}, \delta\right)$. Now for any $d \in \mathcal{C}$, if $c \leqslant d$ then $\delta(c) \subseteq \delta(d)$ so $p_{c} \in \delta(d)$. Conversely, if $p_{c} \in \delta(d)$, then because $p_{c} \notin \operatorname{Prop}\left(D_{c}, \delta\right)$ we must have $d \notin D_{c}$, so $\delta(c) \subseteq \delta(d)$ and by $\delta$-tracking, $c \leqslant d$.

Now for any finite set of formulas $\left\{\varphi_{1}, \ldots, \varphi_{n}\right\}$ a state description is a formula of the form $\pm \varphi_{1} \wedge \cdots \wedge \pm \varphi_{n}$ where $\pm \varphi_{i}$ is either $\varphi_{i}$ or $\neg \varphi_{i}$. We say that a set $\mathrm{C}$ of parameters is $\delta$-finitary iff for each $\mathrm{c} \in \mathrm{C}$ there is a finite set of formula $\varphi_{1}, \ldots, \varphi_{\mathrm{n}} \in \delta(\mathrm{c})$ such that for every $\varphi \in \delta(\mathrm{c})$ and every state description $\sigma$, either $(\sigma \rightarrow \varphi)$ or $(\sigma \rightarrow \neg \varphi)$ is a theorem of $\mathrm{L}(\mathcal{C})$.

The primary examples of $\delta$-finitary sets of parameters are those whose description sets are all finite. But we can get examples of $\delta$-finitary descriptions with infinite description sets from finite models:

LEMMA 15 Suppose $\mathrm{M}$ is a finite $\mathrm{L}(\mathrm{C})$ model and $\mathrm{P}$ is a finite set of propositional variables. For each $\mathrm{c} \in \mathcal{C}$, let $\delta(\mathrm{c})$ be the set of formulas $\varphi$ whose variables are all in $\mathrm{P}$ and which are invariant across c-links, i.e., $\mathrm{M}, \mathrm{u} \models \varphi$ iff $\mathrm{M}, v \models \varphi$, for all $\mathrm{u} \approx_{\mathrm{c}} v$. Then $\mathrm{C}$ is $\delta$-finitary.

Proof: Without loss of generality, assume that Prop $=$ P. Otherwise, we can take a reduct of $M$ to the language based only on propositional variables in $P$. Let $M_{1}, \ldots, M_{n}$ be the modal-equivalence classes of $M$, so that $u, v \in M_{i}$ for some $i$ iff

$$
M, u \models \varphi \text { iff } M, v \models \varphi \text { for all } \varphi \in \mathrm{L}(\mathcal{C})
$$

Then there is a finite sequence $\varphi_{1}, \ldots, \varphi_{n}$ of formulas such that for $u \in M_{i}$,

$$
M, \mathfrak{u} \models \varphi \text { iff }\left(\varphi_{i} \rightarrow \varphi\right) \text { is a theorem of } L(\mathcal{C}) .
$$

The construction of these formulas is a standard technique in modal logic because Prop is finite

Consider $c \in C$. Let $C_{1}, \ldots, C_{m}$ be the $\approx_{c}$-equivalence classes in $M$ and define formulas $\psi_{1}, \ldots, \psi_{m}$ by

\footnotetext{
${ }^{\mathrm{I} 3}$ See, for example, [I, Theorem 32, p. 266].
} 


$$
\psi_{i}=\bigvee\left\{\varphi_{j} \mid M_{j} \subseteq C_{i}\right\}
$$

These formulas are in $\delta(c)$ because they are invariant across $c$-links. Now if $M_{j}$ is not a subset of $C_{i}$ then they are disjoint. (Otherwise, there would be $u, v \in M_{j}$ with $u \in C_{i}$ and $v \notin C_{i}$, so there must be a $\varphi \in \delta(c)$ such that $M, u \models \varphi$ and $M, v \not \models \varphi$, or vice versa, which contradicts $u, v \in M_{i}$.) So

$$
M, u \models \psi_{i} \text { iff } u \in C_{i}
$$

This is because the following are equivalent:

$$
\begin{array}{ll}
M, u \models \psi_{i} & \\
M, u \models \varphi_{j} & \text { for some } M_{j} \subseteq C_{i} \\
u \in M_{j} & \text { for some } M_{j} \subseteq C_{i} \\
{[u] \subseteq C_{i}} & \text { where }[u] \text { is the modal-equivalence class of } u \\
u \in C_{i} & \text { by the disjointness of }[u] \text { and } C_{i} \text { if }[u] \nsubseteq C_{i} .
\end{array}
$$

Now let $\varphi \in \delta(\mathrm{c})$. Then $\varphi$ has a constant value within any $\mathrm{C}_{i}$. Either (I) $M, u \models \varphi$ for each $u \in C_{i}$ or $(2) M, u \models \neg \varphi$ for each $u \in C_{i}$. In case (I), for each $M_{j} \subseteq C_{i}$ and $u \in M_{j}, M, u \models \varphi$, and so $\left(\varphi_{j} \rightarrow \varphi\right)$ is an $L(\mathcal{C})$ theorem. But then $\left(\psi_{i} \rightarrow \varphi\right)$ is also an $\mathrm{L}(\mathcal{C})$ theorem because $\psi_{i}$ is a disjunction of the set of $\varphi_{j}$ for $M_{j} \subseteq C_{i}$. In case (2), by similar reasoning, $\left(\psi_{i} \rightarrow \neg \varphi\right)$ is also an $\mathrm{L}(\mathcal{C})$ theorem. So now either

$$
\left( \pm \psi_{1} \wedge \pm \psi_{m} \rightarrow \varphi\right) \text { or }\left( \pm \psi_{1} \wedge \pm \psi_{m} \rightarrow \neg \varphi\right) \text { is a theorem of } L(\mathcal{C})
$$

This holds for each $c \in \mathcal{C}$ and so $\mathcal{C}$ is $\delta$-finitary.

Next, we need the concept of an 'implicit' parameter. A formula $\varphi$ may not contain parameter $\mathrm{c}$ but it may contain a parameter $\mathrm{d}$ such that $\mathrm{c}$ occurs in a formula of $\delta(d)$. In this case, we say that $c$ occurs implicitly in $\varphi$. More generally, the set of parameters $\mathcal{C}_{\Gamma}$ that occur implicitly in a set of formulas $\Gamma$ are those that occur in formulas of $\Gamma$ or of $\delta(c)$ for some parameter $c$ implicit in $\Gamma$. This, of course, is an inductive definition 14

Now the flexibility of a set of formulas is defined in terms of the description sets for its implicit parameters. We say that $\Gamma$ is $\delta$-flexible iff $\delta=\delta_{1} \cup \delta_{2} \cup \delta_{3}$ such that

I. $\mathcal{C}_{\Gamma}$ is $\delta_{1}$-irreducible

2. $\mathcal{C}_{\Gamma}$ is $\delta_{2}$-finitary

3. $\operatorname{Prop}\left(\mathcal{C}_{\Gamma}, \delta_{1}\right)$ is disjoint from both $\operatorname{Prop}(\Gamma)$ and $\operatorname{Prop}\left(\mathcal{C}_{\Gamma}, \delta_{3}\right)$

Moreover, if $\delta_{2}(\mathrm{c})$ is empty for each $\mathrm{c} \in \mathcal{C}_{\Gamma}$ and $\mathrm{C}_{\Gamma}$ is also $\delta_{1}$ tracking, say that $\Gamma$ is $\delta$-semiflexible. This slightly more rigid kind of description is essential to our main construction.

\footnotetext{
${ }^{14}$ More precisely, $\mathcal{C}_{\Gamma}$ is the smallest subset of $\mathcal{C}$ for which $\Gamma$ and the image of $\mathcal{C}_{\Gamma}$ under $\delta$ are both contained in $\mathrm{L}\left(\mathcal{C}_{\Gamma}\right)$.
} 
So, to recap, for a set of formulas to be flexible, the description of its parameters splits into three parts: the part that only contains propositional variables, which track the $\leqslant$-order, the part that can be eliminated using $\delta$-Expansion because is it basically no more than a finite extension, and the part that is helpfully logically independent of the other parts. Removing the finite extensions takes us back to a semi-flexible core which will form the scaffolding of our model building techniques below.

We ask the reader to forgive this somewhat technical definition until our theorems are proved. After that, we will be returning to $L_{c p}$ and the examples of flexible sets given in the introduction will be fully explained. The main bridge to our result uses sprinkles that witness each modality occurring implicitly in a flexible set of formulas but without interacting with non-sprinkles in an appropriate way.

LEMma I6 If $\Gamma$ is $\delta$-semiflexible then there is a $\delta$-sprinkling of $\mathrm{C}_{\Gamma}$ such that for any $\mathrm{c}, \mathrm{d} \in \mathrm{C}_{\Gamma}$,

I. no sprinkle occurs in $\Gamma$

2. if $\varphi \in \delta(c)$ then either $\varphi$ is a sprinkle or $\varphi$ contains no sprinkle.

3. if $\mathrm{p}_{\mathrm{c}} \in \delta(\mathrm{d})$ then $\mathrm{c} \leqslant \mathrm{d}$.

Proof: Suppose $\Gamma$ is $\delta$-semiflexible. Then $\delta=\delta_{1} \cup \delta_{3}$ such that $\mathcal{C}_{\Gamma}$ is $\delta_{1}$ irreducible and $\delta_{1}$-tracking, and so has a $\delta_{1}$-sprinkling $\mathrm{c} \mapsto \mathrm{p}_{\mathrm{c}}$, by Lemma $\mathrm{I4}$

I. By condition 3 of $\delta$-flexibility, $\operatorname{Prop}(\Gamma)$ and $\operatorname{Prop}\left(\mathcal{C}_{\Gamma}, \delta_{1}\right)$ are distinct and since the latter contains all the sprinkles, no sprinkle occurs in $\Gamma$.

2. Suppose $\varphi \in \delta(d)$ for some $d \in \mathcal{C}_{\Gamma}$. Then $\varphi$ is either in $\delta_{1}(d)$ or in $\delta_{3}(d)$. In the first case, $\varphi$ must be a propositional variable, so if it contains a sprinkle it just is that sprinkle. In the second case, any propositional variable it contains must be in $\operatorname{Prop}\left(\mathrm{C}_{\Gamma}, \delta_{3}\right)$, which is disjoint from the sprinkle-containing $\operatorname{Prop}\left(\mathrm{C}_{\Gamma}, \delta_{1}\right)$, condition 3 of the definition of flexibility, and so it can contain no sprinkle.

3. Suppose $p_{c} \in \delta(d)$ for some $d \in \mathcal{C}_{\Gamma}$. Then $p_{c}$ is either in $\delta_{1}(d)$ or in $\delta_{3}(\mathrm{~d})$. But for the reason just mentioned, $\delta_{3}(\mathrm{~d})$ cannot contain a sprinkle and so $p_{c} \in \delta_{1}(d)$. So by the definition of $\delta_{1}$-sprinkling, $c \leqslant d$.

The lemma tells us that we can work with a set of propositional variablesthe sprinkles - that encode the ordering of parameters without interacting with $\Gamma$ or the other formulas in the description sets. This is enough to build $\delta$-models, as the following result shows. 
LEMMA 17 Given a well-founded description $\delta$ for $\mathcal{C}$, every $\delta$-semiflexible set $\Gamma$ of $\mathrm{L}(\mathcal{C})$ formula is satisfied by a $\delta$-model if it is consistent with $\delta$-Inclusion and $\delta$-Preservation together with the axioms and rules of normal modal logic. ${ }^{15}$

Proof: Suppose $\Gamma$ is $\delta$-semiflexible and consistent. Then by Lemma 16 there is a sprinkle $p_{c}$ for each $c \in \mathcal{C}_{\Gamma}$ for which $\delta(c) \neq \emptyset$. By Theorem 4 , there is a model, $M=\langle W, R, \approx, V\rangle$ and $w_{0} \in W$ such that $M, w_{0} \models \Gamma$. Our strategy will be as follows:

I. Define a model $M^{\prime}=\left\langle W, R, \approx, V^{\prime}\right\rangle$ in which the valuation function is changed, from $V$ to $V^{\prime}$, only for the sprinkles, so the distribution of each sprinkle $p_{c}$ appropriately encodes the equivalence relation $\approx_{c}$; specifically, when Ruv,

$$
u \approx_{c} v \text { iff }\left(u \in V^{\prime}\left(p_{c}\right) \text { iff } v \in V^{\prime}\left(p_{c}\right)\right)
$$

2. Show that $\Gamma$ is satisfied by the $\delta$-model $\left[\delta^{\infty}\right] M^{\prime}$ at $\left.w_{0}\right]^{16}$

For the first stage, we define $V^{\prime}$ by partitioning the set $W$ of states. From the proof of Theorem 4, we may assume that $R$ is a tree with root $w_{0}$. In other words, each state $w$ other than $w_{0}$ has a mother $\hat{w}$, being the unique state for which R $\hat{w} w$. We can partition the states of $W$ into generations, starting at the root: $W_{0}=\left\{w_{0}\right\}$ and $W_{n+1}=\left\{w \in W \mid \hat{w} \in W_{n}\right\}$. Thus $W_{n}$ is the set of states exactly $n$ transitions along $R$ from $w_{0}$. Now for each sprinkle $p_{c}$, let

$$
\begin{array}{lll}
V_{0}\left(p_{c}\right) & =\left\{w_{0}\right\} \\
V_{n+1}\left(p_{c}\right)=V_{n}\left(p_{c}\right) & \cup\left\{w \in W_{n+1} \mid \hat{w} \in V_{n}\left(p_{c}\right) \text { and } \hat{w} \approx_{c} w\right\} \\
& \cup\left\{w \in W_{n+1} \mid \hat{w} \notin V_{n}\left(p_{c}\right) \text { and } \hat{w} \not_{c} w\right\}
\end{array}
$$

And then let

$$
V^{\prime}(p)= \begin{cases}\bigcup_{n} V_{n}\left(p_{c}\right) & \text { if } p=p_{c} \text { for some } c \in \mathcal{C}_{\Gamma} \\ V(p) & \text { otherwise }\end{cases}
$$

The construction ensures precisely that if Ruv then for each sprinkle $p_{c}$,

$$
\left.{ }^{*}\right) \quad u \approx_{c} v \text { iff }\left(u \in V^{\prime}\left(p_{c}\right) \text { iff } v \in V^{\prime}\left(p_{c}\right)\right)
$$

For the second stage, note that $M$ and $M^{\prime}$ agree on the formulas of $\Gamma$ because they differ only on the valuation of sprinkles, which do not occur in $\Gamma$. So by Lemma $2, M^{\prime}, w_{0} \models \Gamma$. Next, we must show that the $[\delta]$ operation produces only models that also agree on $\Gamma$. $[\delta]$ does not change the valuation, so all we need to check is that for each $\alpha$

(**) for all $\mathrm{c} \in \mathcal{C}_{\Gamma}, \mathrm{R} \cap \approx_{\mathrm{c}}=\mathrm{R} \cap \approx_{\left[\delta^{\alpha}\right] \mathrm{c}}$

\footnotetext{
${ }^{15}$ We only need $\delta$-Inclusion rather than the full power of $\delta$-Expansion here. The latter will be used in the next lemma.

${ }^{16}$ Where $\left[\delta^{\infty}\right]$ is the construction used in Lemma
} 
From $(* *)$, we get that that $M^{\prime}$ and $\left[\delta^{\infty}\right] M^{\prime}$ agree on $\Gamma$. This is because they have the same valuation, $V^{\prime}$, and for every parameter $c$ occurring in $\Gamma$, if $\alpha$ is the depth of $c$, then $\approx_{\left[\delta^{\infty}\right] c}=\approx_{\left[\delta^{\alpha+1}\right] c}$, by definition of $\delta^{\infty}$. This in turns implies that $R \cap \approx_{c}=R \cap \approx_{\left[\delta^{\infty}\right] c}$, by $\left.{ }^{* *}\right)$. Then, by Lemma $2,\left[\delta^{\infty}\right] M^{\prime}, w_{0} \models \Gamma$.

For the proof of $\left({ }^{* *}\right)$, we proceed by induction on $\alpha$. The case in which $\alpha=0$ is trivial, since $\left[\delta^{0}\right] M^{\prime}=M^{\prime}$. For the successor case, suppose $\alpha=\beta+1$, $c \in \mathcal{C}_{\Gamma}$ and Ruv. We must show that:

$$
u \approx_{c} v \text { iff } u \approx_{[\delta \beta+1] c} v
$$

First the left-to-right direction. Suppose that $u \approx_{c} v$ and $\varphi \in \delta(c)$. We must show that $\left[\delta^{\beta}\right] M^{\prime}, u \models \varphi$ iff $\left[\delta^{\beta}\right] M^{\prime}, v \models \varphi$. There are two cases, depending on whether or not $\varphi$ is a sprinkle.

Case (i): $\varphi$ is the sprinkle $p_{d}$ for some $d \in \mathcal{C}_{\Gamma}$. But then $p_{d} \in \delta(c)$ and so $\mathrm{d} \leqslant \mathrm{c}$, by Lemma $\mathrm{I} 6$ part 3 . So by the basic constraint on $\mathrm{L}(\mathrm{C})$-models, $u \approx_{d} v$. Then by $\left(^{*}\right), u \in V^{\prime}\left(p_{d}\right)$ iff $v \in V^{\prime}\left(p_{d}\right)$, and so $\left[\delta^{\beta}\right] M^{\prime}, u \models p_{d}$ iff $\left[\delta^{\beta}\right] M^{\prime}, v \models p_{d}$, because $M^{\prime}$ and $\left[\delta^{\beta}\right] M^{\prime}$ have the same valuation.

Case (ii): $\varphi$ is not a sprinkle. Then $\varphi$ does not even contain a sprinkle, by Lemma I6 part 2. So $V(p)=V^{\prime}(p)$ for each propositional variable $p$ occurring in $\varphi$, and for all parameters $\mathrm{d}$ occurring in $\varphi$, by inductive hypothesis, $\mathrm{R} \cap \approx_{\mathrm{d}}$ $\left.=\mathrm{R} \cap \approx_{\left[\delta^{\beta}\right] \mathrm{d}}\right]^{17}$ Hence $M$ and $\left[\delta^{\beta}\right] M^{\prime}$ agree on $\varphi$, and so by Lemma $2, M, w \models$ $\varphi$ iff $\left[\delta^{\beta}\right] M^{\prime}, w \models \varphi$ for each $w \in W$. Finally, $\delta$-Preservation ensures that $M, \mathfrak{u} \models \varphi$ iff $M, v \models \varphi$, and so $\left[\delta^{\beta}\right] M^{\prime}, u \models \varphi$ iff $\left[\delta^{\beta}\right] M^{\prime}, v \models \varphi$, as required.

Now for the right-to-left direction. Suppose $u \approx_{\left[\delta^{\beta+1}\right] c} v$. There are two cases, depending on whether $\mathrm{c}$ is a least element of $\mathrm{C}_{\Gamma}$.

Case (i): $c$ is a least element. Since $R$ in $M^{\prime}$ is defined as the union of the $R_{c}$ 's, and because we already assumed that $R u v$, it must be that $R_{d} u v$ for some d. But $c \leqslant d$ because $c$ is a least element, and so $u \approx_{c} v$.

Case (ii): $c$ is a non-least element. Then it has a sprinkle $p_{c} \in \delta(c)$, so $\left[\delta^{\beta}\right] M^{\prime}, u \models p_{c}$ iff $\left[\delta^{\beta}\right] M^{\prime}, v \models p_{c}$, so $u \in V^{\prime}\left(p_{c}\right)$ iff $v \in V^{\prime}\left(p_{c}\right)$, so $u \approx_{c} v$ by $(*)$.

Finally, for the case in which $\alpha$ is a limit ordinal,

$$
\approx_{\left[\delta^{\alpha}\right] \mathrm{c}}= \begin{cases}\approx_{\left[\delta^{\gamma+1}\right] \mathrm{c}} & \text { if } \mathrm{c} \text { has depth } \gamma<\alpha \\ \approx_{\mathrm{c}} & \text { otherwise }\end{cases}
$$

But $\gamma<\alpha$ and $\alpha$ is a limit ordinal, so $\gamma+1<\alpha$, and so by inductive hypothesis, $\mathrm{R} \cap \approx_{\mathrm{c}}=\mathrm{R} \cap \approx_{\left[\delta^{\gamma+1}\right] \mathrm{c}}$ and we are done.

We can now extend this result to $\delta$-flexible sets to get our main theorem. THEOREM I8 If $\delta$ is well-founded then every $\mathrm{L}(\mathcal{C})_{\delta}$-consistent $\delta$-flexible set has a $\delta$ model.

\footnotetext{
${ }^{17}$ It is here that we use the fact that $\mathcal{C}_{\Gamma}$ contains all of the parameters implicit in $\Gamma$, and not just those that occur explicitly in $\Gamma$.
} 
Proof: Suppose $\Gamma$ is $\mathrm{L}(\mathcal{C})_{\delta}$-consistent and $\delta$-flexible. Then $\delta=\delta_{1} \cup \delta_{2} \cup \delta_{3}$ such that

I. $\mathcal{C}_{\Gamma}$ is $\delta_{1}$-irreducible

2. $\mathcal{C}_{\Gamma}$ is $\delta_{2}$-finitary

3. $\operatorname{Prop}\left(\mathfrak{C}_{\Gamma}, \delta_{1}\right)$ is disjoint from both $\operatorname{Prop}(\Gamma)$ and $\operatorname{Prop}\left(\mathcal{C}_{\Gamma}, \delta_{3}\right)$

We need to subtract the $\delta_{2}$ part from the description of each parameter. In languages such as $\mathrm{L}_{\mathfrak{c} p}$ this is straightforward because it contains ceteris paribus modalities for every set of formulas. But in the general case, we will need to add some parameters to possess these stripped-down descriptions. So first make a copy of $\mathcal{C}: \mathrm{C}^{-}=\left\{\mathrm{c}^{-} \mid \mathrm{c} \in \mathcal{C}\right\}$ where the $\mathrm{c}^{-}$are new parameters with $\mathrm{c}^{-} \leqslant \mathrm{d}^{-}$ iff $\delta_{1}(\mathrm{c}) \subseteq \delta_{1}(\mathrm{~d})$, so that $\mathrm{C}^{-}$is $\delta_{1}$-tracking. Define $\delta^{-}\left(\mathrm{c}^{-}\right)=\delta_{1}(\mathrm{c}) \cup \delta_{3}(\mathrm{c})$. In other words, each new parameter $\mathrm{c}^{-}$is associated with those formulas in the description of $c$ that are not in the finitary part. Now expand to $\mathcal{C}^{\dagger}=\mathcal{C} \cup \mathcal{C}^{-}$ with $c^{-} \leqslant d$ iff $c \leqslant d$ and $\leqslant$ preserved within $\mathcal{C}$ and $\mathcal{C}^{-}$and define $\delta^{\dagger}$ to be $\delta \cup \delta^{-}{ }^{18}$ Note in particular that $\delta^{\dagger}\left(c^{-}\right) \subseteq \delta^{\dagger}(\mathrm{c})$ and $\delta_{2}(\mathrm{c})=\delta^{\dagger}(\mathrm{c}) \backslash \delta^{\dagger}\left(\mathrm{c}^{-}\right)$.

Let $c$ be any parameter that occurs implicitly in $\Gamma$. It is $\delta_{2}$-finitary and so there is a finite set of formula $\varphi_{1}, \ldots, \varphi_{n} \in \delta_{2}$ (c) such that for every $\varphi \in \delta_{2}$ (c) and every state description $\sigma$ either $(\sigma \rightarrow \varphi)$ or $(\sigma \rightarrow \neg \varphi)$ is a theorem of $\mathrm{L}(\mathcal{C})_{\delta_{2}}$. Let $\Sigma_{\mathrm{c}}$ be the set of those state description, then for each $\sigma \in \Sigma$, the following are all theorems of $\mathrm{L}(\mathcal{C})_{\delta^{\dagger}}$ :
(I) $\quad(\sigma \rightarrow[c] \sigma)$
(2) $\quad(\sigma \wedge\langle c\rangle \psi) \rightarrow(\sigma \wedge\langle c\rangle(\sigma \wedge \psi))$
by $\delta_{2}$-Preservation
(3) $\quad(\sigma \wedge\langle c\rangle(\sigma \wedge \psi)) \rightarrow\left(\sigma \wedge\left\langle c^{-}\right\rangle(\sigma \wedge \psi)\right)$
from (I) by normal modal logic
(4) $\quad(\sigma \wedge\langle c\rangle \psi) \rightarrow\left(\sigma \wedge\left\langle c^{-}\right\rangle(\sigma \wedge \psi)\right)$
by $\delta^{\dagger}$-Inclusion and modal logic
because $\delta^{\dagger}\left(\mathrm{c}^{-}\right) \subseteq \delta^{\dagger}(\mathrm{c})$
(5) $\bigvee\left\{\sigma \mid \sigma \in \Sigma_{c}\right\}$
(6) $\langle\mathrm{c}\rangle \psi \leftrightarrow \bigvee_{\sigma \in \Sigma_{\mathrm{c}}}(\sigma \wedge\langle\mathrm{c}\rangle \psi)$
(7) $\langle c\rangle \psi \rightarrow \bigvee_{\sigma \in \Sigma_{c}}\left(\sigma \wedge\left\langle c^{-}\right\rangle(\sigma \wedge \psi)\right)$
from (2) and (3)
by propositional logic
from (5) by propositional logic
from (4) and (6) and
propositional logic
(8) $\sigma \rightarrow \varphi$ or $\sigma \rightarrow \neg \varphi$
(9) $\quad\left(\sigma \wedge\left\langle c^{-}\right\rangle(\sigma \wedge \psi)\right) \rightarrow\langle c\rangle \psi$ for each $\varphi \in \delta_{2}(\mathrm{c})$ by $\delta_{2}$-finitary
(Io) $\bigvee_{\sigma \in \Sigma_{c}}\left(\sigma \wedge\left\langle c^{-}\right\rangle(\sigma \wedge \psi)\right) \rightarrow\langle c\rangle \psi$
(II) $\langle\mathrm{c}\rangle \psi \leftrightarrow \bigvee_{\sigma \in \Sigma_{\mathrm{c}}}\left(\sigma \wedge\left\langle\mathrm{c}^{-}\right\rangle(\sigma \wedge \psi)\right)$ from (8) by $\delta^{\dagger}$-Expansion because $\delta_{2}(\mathrm{c})=\delta^{\dagger}(\mathrm{c}) \backslash \delta^{\dagger}\left(\mathrm{c}^{-}\right)$ from (9) by propositional logic from (7) and (IO)

Now let $\psi^{c}$ be the formula $\bigvee_{\sigma \in \Sigma_{c}}\left(\sigma \wedge\langle c\rangle^{-}(\sigma \wedge \psi)\right)$. We have shown that $\langle c\rangle \psi \leftrightarrow \psi^{\mathrm{c}}$ is a theorem of $\mathrm{L}(\mathrm{C})_{\delta^{\dagger}}$. This can now be used to eliminate the

\footnotetext{
${ }^{18}$ This is a description because the $\mathcal{C}$ and $\mathcal{C}^{-}$are disjoint and $\delta^{-}$satisfies the defining condition for descriptions on each part, and moreover, if $c^{-} \leqslant d$ then $c \leqslant d$ so $\delta^{\dagger}\left(c^{-}\right)=\delta^{-}\left(c^{-}\right)=$ $\delta_{1}(\mathrm{c}) \cup \delta_{3}(\mathrm{c}) \subseteq \delta(\mathrm{c}) \subseteq \delta(\mathrm{d})=\delta^{\dagger}(\mathrm{d})$
} 
occurrences of any $c \in C_{\Gamma}$ from any formula in favour of $c^{-}$. Let $\varphi^{-}$be the result of systematically replacing occurrences of $\langle c\rangle \psi$ by $\psi^{\mathrm{c}}$, for each $c \in \mathrm{C}_{\Gamma}{ }^{19}$ It then follows that $\varphi \leftrightarrow \varphi^{-}$is also a theorem of $\mathrm{L}(\mathcal{C})_{\delta^{\dagger}}$.

Let $\Gamma^{-}=\left\{\varphi^{-} \mid \varphi \in \Gamma\right\}$. Now $\Gamma$ is $\delta$-consistent and so $\delta^{\dagger}$-consistent, because $\delta$ and $\delta^{\dagger}$ agree on all the parameters implicit in $\Gamma$, which are all in $\mathcal{C}$. And since $\Gamma^{-}$is obtained from $\Gamma$ only by replacing logical equivalents, it is also $\mathrm{L}(\mathrm{C})_{\delta^{\dagger}}$ consistent. Moreover, by Lemma I2 if $M$ is a $\delta^{\dagger}$-model and $w$ is in the domain of $M$ then

$$
M, w \models \Gamma \text { iff } M, w \models \Gamma^{-}
$$

Moreover, from any $\delta^{\dagger}$-model $M$ we can get a $\delta$-model $M^{\prime}$ just by forgetting the interpretation of parameters in $\mathrm{C}^{-}$. This model will satisfy the same formulas of $\mathrm{L}(\mathcal{C})$ at the same points. And, finally, $\Gamma^{-}$, all of whose implicit parameters are in $\mathrm{C}^{-}$, is $\delta^{\dagger}$-semiflexible. So by Lemma $\mathrm{I} 7, \Gamma^{-}$is satisfied by a $\delta^{\dagger}$-model $M$ at some point $w$, and hence the $\delta$-model $M^{\prime}$ also satisfies $\Gamma$ at $w$.

\section{3 ВАCK TO $\mathrm{L}_{\mathrm{cp}}$}

The above results about parametric ceteris paribus logic have consequences for the fully explicit language $L_{c p}$. To make the connection, recall that $L_{c p}$ is a special case of $L(\mathcal{C})$ in which $\mathcal{C}$ is the collection of subsets of $L_{c p}$ ordered by $\Gamma_{1} \leqslant \Gamma_{2}$ iff $\Gamma_{2} \subseteq \Gamma_{1}$. Since this makes $\mathcal{C}$ a proper class, we will typically restrict our attention to a suitable set-sized fragment of $L(\mathcal{C})$. For example, when looking for models satisfying a set $\Gamma$ of $L(\mathcal{C})$ formulas, it is sufficient to consider the language $\mathrm{L}\left(\mathcal{C}_{\Gamma}\right)$, which contains $\Gamma$. The set $\mathcal{C}_{\Gamma}$ is just the set of those $\Delta$ which occur as $\langle\Delta\rangle$ in some formula of $\Gamma$, since in $L_{c p}$, any implicit occurrence is also an explicit occurrence.

With this definition of $\mathcal{C}$ and taking $\delta$ to be the identity function, we get the following axioms for $L_{c p}$ :

$$
\begin{array}{ll}
\text { (CP-Preservation) } & \vdash \varphi \rightarrow[\Gamma] \varphi \text { and }\langle\Gamma\rangle \varphi \rightarrow \varphi \text { for all } \varphi \in \Gamma . \\
\text { (CP-Expansion) } & \text { If } \vdash(\theta \rightarrow \varphi) \text { or } \vdash(\theta \rightarrow \neg \varphi) \text { for each } \varphi \in \Gamma \backslash \Gamma^{\prime} \\
& \text { then } \vdash\left(\theta \wedge\left\langle\Gamma^{\prime}\right\rangle(\theta \wedge \psi)\right) \rightarrow\langle\Gamma\rangle \psi
\end{array}
$$

Call the logic obtained by adding these to the axioms and rules of normal modal logic, LCP. As before the inclusion principle is derivable:

\begin{aligned} (CP-Inclusion) $\quad & \vdash\langle\Gamma\rangle \rightarrow\left\langle\Gamma^{\prime}\right\rangle \varphi$ for each $\Gamma^{\prime} \subseteq \Gamma \\$\hline${ }^{\text {I9 }}$ Specifically, $[p]^{-} & =p \\ {[-\varphi]^{-} } & =-[\varphi]^{-} \\ {[(\varphi \wedge \psi)]^{-} } & =\left[\left(\varphi^{-} \wedge \psi^{-}\right)\right] \\ {[\langle c\rangle \psi]^{-} } & = \begin{cases}\psi^{\mathrm{c}} & \text { if } c \in \mathrm{C}_{\Gamma} \\ \langle c\rangle[\psi]^{-} & \text {otherwise }\end{cases} \end{aligned}$


LCP is an extension of the axiomatisation of the finite fragment of $\mathrm{L}_{\mathfrak{c p}}$ given by [4] (and discussed in Section I] because all formulas of the form

$$
\langle\psi, \Gamma\rangle \varphi \quad \leftrightarrow \quad(\psi \wedge\langle\Gamma\rangle(\psi \wedge \varphi)) \vee(\neg \psi \wedge\langle\Gamma\rangle(\neg \psi \wedge \varphi))
$$

are derivable 20

The correspondence between $\mathrm{L}_{c p}$ with the logic LCP and $\mathrm{L}(\mathrm{C})$ with the logic $\mathrm{L}(\mathcal{C})_{\delta}$ at both the syntactic and semantic levels can now be noted with the following two lemmas.

LEMMA 19 A set of formulas $\Gamma$ is $\mathrm{LCP}$-consistent iff it is $\mathrm{L}\left(\mathrm{C}_{\Gamma}\right)_{\delta}$-consistent, when $\delta$ is the identity function.

Proof: By definition of the axioms.

LEMma 20 A set $\Gamma$ of $\mathrm{L}_{\mathrm{cp}}$ formulas is satisfiable (in an $\mathrm{L}_{\mathrm{cp}}$ model) iff it is satisfiable in an $\mathrm{L}\left(\mathcal{C}_{\Gamma}\right) \delta$-model, where $\delta$ is the identity function.

Proof: If the $\mathrm{L}\left(\mathcal{C}_{\Gamma}\right) \delta$-model $M=\langle\mathrm{W}, \mathrm{R}, \approx, \mathrm{V}\rangle$ satisfies $\Gamma$ then the $\mathrm{L}_{\mathrm{cp}}$ model $M^{\prime}=\langle W, R, V\rangle$ also satisfies $\Gamma$, as the relation $\approx_{\Delta}$ in both models coincides for $\Delta \in \mathcal{C}_{\Gamma}$. Specifically, the following are equivalent:

$$
\begin{array}{ll}
u & \approx_{\Delta} v \text { in } M \\
\text { for each } \varphi \in \delta(\Delta), M, u \models \varphi \text { iff } M, v \models \varphi & M \text { is a } \delta \text {-model } \\
\text { for each } \varphi \in \Delta, M, \mathfrak{u} \models \varphi \text { iff } M, v \models \varphi & \delta \text { is the identity function } \\
\mathfrak{u} \approx_{\Delta} v \text { in } M^{\prime} & \text { definition in } M^{\prime}
\end{array}
$$

Conversely, given any $L_{c p}$ model $M=\langle W, R, V\rangle$ that satisfies $\Gamma$, let $M^{\prime}$ be the $\mathrm{L}(\mathcal{C})$ model $\langle W, R, \approx, V\rangle$ with $\approx$ defined in the $\mathrm{L}_{\mathrm{cp}}$ way: $u \approx_{\Gamma} v$ iff for each $\varphi \in \Gamma, M, u \models \varphi$ iff $M, v \models \varphi$. Again, for similar reasons, $M^{\prime}$ is a $\delta$-model and satisfies $\Gamma$.

We can now specialise the concept of $\delta$-flexibility as follows ${ }^{21}$

\footnotetext{
${ }^{20} \mathrm{CP}$-Inclusion and CP-Preservation are used to go from left to right and CP-Expansion is used from right to left.

${ }^{21}$ Notice that we do not need anything explicit relating to $\delta$-tracking, as the $\leqslant$ order is given by $\subseteq$ in $\mathrm{L}_{\mathrm{cp}}$, so that $\Gamma_{1} \leqslant \Gamma_{2}$ iff $\Gamma_{1} \subseteq \Gamma_{2}$.
} 
Definition of Flexibility: A set $\Gamma$ of $\mathrm{L}_{\mathrm{cp}}$ formulas is flexible iff each modality $\langle\Delta\rangle$ occurring in $\Gamma$ is of the form $\left\langle\Delta_{1}, \Delta_{2}, \Delta_{3}\right\rangle$ where

I. $\Delta_{1}$ is irreducible: $\Delta_{1} \subseteq$ Prop and if

$$
\Delta_{1} \subseteq \bigcup_{\Delta^{\prime} \in \mathrm{D}} \Delta_{1}^{\prime}
$$

and $\left\langle\Delta^{\prime}\right\rangle$ occurs in $\Gamma$ for each $\Delta^{\prime} \in \mathrm{D}$, then $\Delta_{1} \subseteq \Delta_{1}^{\prime}$ for some $\Delta^{\prime} \in \mathrm{D}$

2. $\Delta_{1}$ tracks the order of $\Delta \backslash \Delta_{2}$ :

$$
\Delta \backslash \Delta_{2} \subseteq \Delta^{\prime} \backslash \Delta_{2}^{\prime} \quad \text { iff } \quad \Delta_{1} \subseteq \Delta_{1}^{\prime}
$$

3. $\Delta_{2}$ is finitary: there is a finite set $\left\{\varphi_{1}, \ldots, \varphi_{n}\right\} \subseteq \Delta$ such that for each $\varphi \in \Delta_{2}$

$$
\text { either }\left( \pm \varphi_{1} \wedge \cdots \wedge \pm \varphi_{n}\right) \rightarrow \varphi \text { or }\left( \pm \varphi_{1} \wedge \cdots \wedge \pm \varphi_{n}\right) \rightarrow \neg \varphi
$$

is a tautology, where $\pm \varphi_{i}$ is either $\varphi_{i}$ or $\neg \varphi_{i}$.

4. none of the propositional variables in $\Delta_{1}$ occurs in $\Delta_{3}$ or in any formula of $\Gamma$ except within a modal operator.

Checking definitions, it can easily be verified that LEMMA 2I A set $\Gamma$ of $\mathrm{L}_{\mathrm{cp}}$ formulas is flexible iff $\Gamma$ is $\delta$-flexible (as a set of $\mathrm{L}\left(\mathcal{C}_{\Gamma}\right)$ formulas) with $\delta$ the identity function.

And with this, we can prove the following as a special case of Theorem 18 . THEOREM 22 Every flexible and LCP-consistent set of formulas is satisfiable.

Proof: Suppose $\Gamma$ is flexible and LCP-consistent. Then by Lemma $2 \mathrm{I}$ it is also $\delta$-flexible and by Lemma 19 it is $L\left(\mathcal{C}_{\Gamma}\right)_{\delta}$-consistent, where $\delta$ is the identity function. We also need to know that $\delta$ is well-founded, i.e. that $\mathcal{C}=\bigcup_{n} \mathcal{C}_{n}$. This follows from the inductive definition of $L_{c p}$ itself. So, by Theorem 18 , $\Gamma$ is satisfiable in an $L\left(\mathcal{C}_{\Gamma}\right) \delta$-model. Finally, by Lemma 20 , $\Gamma$ is satisfiable in an $L_{c p}$ model.

From a different perspective, we can see an $L_{c p}$ formula as the result of applying a description to a parametric formula. Indeed, for a well-founded description $\delta$ for a condition set $\mathcal{C}$, we can demonstrate an equivalence between the logic of $\mathrm{L}(\mathrm{e})$ and the fragment of $\mathrm{L}_{c p}$ obtained by iteratively replacing each symbol $\mathrm{c}$ by $\delta(\mathrm{c})$.

To make this precise, let $\mathrm{L}_{\mathfrak{c p}}(\mathrm{C})$ be the language of propositional modal logic with propositional variables Prop, whose modalities are of the form $\langle c\rangle$ 
for $c \in \mathcal{C}$ and $\langle\Gamma\rangle$ for $\Gamma \subseteq \mathrm{L}_{\mathrm{cp}}(\mathcal{C})$. Note that this language contains both $\mathrm{L}(\mathcal{C})$ and $\mathrm{L}_{c p}$. The semantics for this language is the obvious combination of its components. We take an $\mathrm{L}(\mathrm{C})$ model $M=\langle\mathrm{W}, \mathrm{R}, \approx, \mathrm{V}\rangle$ and define $\approx_{\Gamma}$ in the usual way: $\mathfrak{u} \approx_{\Gamma} v$ iff for each $\varphi \in \Gamma, M, u \models \varphi$ iff $M, v \models \varphi$. Then define

$$
\begin{array}{lll}
M, u \models\langle c\rangle \varphi & \text { iff } & \text { Ruv and } u \approx_{c} v \text { and } M, v \models \varphi \text { for some } v \in W \\
M, u \models\langle\Gamma\rangle \varphi & \text { iff } & R u v \text { and } u \approx_{\Gamma} v \text { and } M, v \models \varphi \text { for some } v \in W
\end{array}
$$

Note that if $\varphi$ is an $L_{c p}$ formula then $M, u \models \varphi$ iff $M^{\prime}, u \models \varphi$, where $M^{\prime}$ is the $\mathrm{L}_{\mathrm{cp}} \operatorname{model}\langle\mathrm{W}, \mathrm{R}, \mathrm{V}\rangle$.

Given a description $\delta$ of $\mathrm{L}(\mathcal{C})$, let:

$$
\begin{array}{ll}
\delta^{\alpha}(\mathrm{c}) & =\{\varphi[\delta, \alpha] \mid \varphi \in \delta(\mathrm{c})\} \\
\varphi[\delta, \alpha] & =\text { result of replacing in } \varphi \text { all parameters } \mathrm{c} \text { of depth } \beta<\alpha \text { by } \delta^{\beta}(\mathrm{c}) \\
\varphi[\delta, \infty] & =\text { result of replacing in } \varphi \text { all parameters } \mathrm{c} \text { of depth } \alpha \text { by } \delta^{\alpha}(\mathrm{c}) .
\end{array}
$$

So $\delta^{0}(\mathrm{c})=\delta(\mathrm{c}), \delta^{1}(\mathrm{c})$ is the result of replacing in $\varphi$ all parameters $\mathrm{c}$ of depth o by $\delta(c), \delta^{\omega}(c)$ is the result of replacing in $\varphi$ all parameters $c$ of depth $n$ by $\delta^{n}(c)$, and so on. The satisfaction of formulas of sufficiently low depth is coordinated between models $M$ and $\left[\delta^{\alpha}\right] M$ in the following manner:

LEMMA 23 For a formula $\varphi$ of depth $\leqslant \alpha$,

$$
\left[\delta^{\alpha}\right] M, w \models \varphi \text { iff } M, u \models \varphi[\delta, \alpha]
$$

Proof: By induction (i) on $\alpha$ and then (ii) on $\varphi$. For the base cases, note that $\left[\delta^{0}\right] M=M$ and $\left[\delta^{\alpha}\right] M$ and $M$ agree on propositional variables. For the second induction, the only interesting case is when $\varphi$ has the form $\langle c\rangle \psi$.

Let $\beta$ be the depth of $\mathrm{c}$. Then $\beta<\alpha$, so by Lemma $\mathrm{IO} \approx_{\left[\delta^{\alpha}\right] \mathrm{c}}=\approx_{\left[\delta^{\beta+1}\right] \mathrm{c}}$.

Thus, $\left[\delta^{\alpha}\right] M, w \models\langle c\rangle \psi$ iff $\left[\delta^{\alpha}\right] M, v \models \psi$ for some $v$ such that Rwv and $w \approx_{[\delta \beta+1] c} v$

iff $\left[\delta^{\alpha}\right] M, v \models \psi$ for some $v$ such that Rwv and $\left[\delta^{\beta}\right] M, w \models \theta$ iff $\left[\delta^{\beta}\right] M, v \models \theta$ for all $\theta \in \delta(c)$, by definition of $\approx_{\left[\delta^{\beta+1}\right] \mathrm{c}}$.

For $\theta \in \delta(c)$, the depth of $\theta \leqslant \beta<\alpha$, so $\left[\delta^{\beta}\right] M, u \models \theta$ iff $M, u \models \theta[\delta, \beta]$, by induction hypothesis (i).

Hence, $\left[\delta^{\alpha}\right] M, w \models\langle c\rangle \psi$ iff $\left[\delta^{\alpha}\right] M, v \models \psi$ for some $v$ such that Rwv and $M, w \models \theta[\delta, \beta]$ iff $M, v \models \theta[\delta, \beta]$ for all $\theta \in \delta(c)$.

But $\delta^{\beta}(\mathrm{c})=\{\theta[\delta, \beta] \mid \theta \in \delta(\mathrm{c})\}$.

Hence, $\left[\delta^{\alpha}\right] M, w \models\langle c\rangle \psi$ iff $\left[\delta^{\alpha}\right] M, v \models \psi$ for some $v$ such that Rwv and $M, w \models \chi$ iff $M, v \models \chi$ for all $\chi \in \delta^{\beta}(c)$.

But since $\beta<\alpha, \theta[\delta, \beta]=\theta[\delta, \alpha]$, by definition, so $\delta^{\beta}(\mathrm{c})=\delta^{\alpha}(\mathrm{c})$, also by definition. 
So, $\quad\left[\delta^{\alpha}\right] M, w \models\langle c\rangle \psi \quad$ iff $\quad\left[\delta^{\alpha}\right] M, v \models \psi$ for some $v$ such that Rwv and $M, w \models \chi$ iff $M, v \models \chi$ for all $\chi \in \delta^{\alpha}(\mathrm{c})$.

iff $M, v \models \psi[\delta, \alpha]$ for some $v$ such that Rwv and $M, w \models \chi$ iff $M, v \models \chi$ for all $\chi \in \delta^{\alpha}(\mathrm{c})$, by inductive hypothesis (ii).

iff $M, w \models\left\langle\delta^{\alpha}(c)\right\rangle \psi[\delta, \alpha]$ by semantic definition.

iff $M, w \models(\langle c\rangle \psi)[\delta, \alpha]$, because the depth of $c=\beta<\alpha$.

Now, for any description $\delta$ and any formula $\varphi$ in $\mathrm{L}(\mathcal{C}), \varphi[\delta, \infty]$ is a formula of $\mathrm{L}_{\mathrm{cp}}$. This establishes the following interpretation of $\mathrm{L}(\mathcal{C})$ into $\mathrm{L}_{\mathfrak{c} p}$ :

LEMMA $24\left[\delta^{\infty}\right] M, w \models \varphi$ iff $M, u \models \varphi[\delta, \infty]$

Proof: Suppose $\varphi$ has depth $\alpha$. Then any parameter c occurring in $\varphi$ has depth $\beta<\alpha$. By definition, $\approx_{\left[\delta^{\infty}\right] \mathrm{c}}=\approx_{\left[\delta^{\beta+1}\right] \mathrm{c}}$ and by Lemma $\mathrm{IO}, \approx_{\left[\delta^{\beta+1}\right] \mathrm{c}}=\approx_{\left[\delta^{\alpha}\right] \mathrm{c}}$, so by Lemma 2 .

$$
\left[\delta^{\infty}\right] M, w \models \varphi \text { iff }\left[\delta^{\alpha}\right] M, w \models \varphi
$$

And by Lemma 23 .

$$
\left[\delta^{\alpha}\right] M, w \models \varphi \text { iff } M, u \models \varphi[\delta, \alpha]
$$

But $\varphi[\delta, \infty]=\varphi[\delta, \alpha]$ (since $\varphi$ has depth $\alpha$ ) and we are done.

The logic of $\mathrm{L}(\mathcal{C})$ over $\delta$-models is therefore strongly related to the fragment of $L_{c p}$ given by the image under $\varphi[\delta, \alpha]$. A set of $L(\mathcal{C})$-formulas $\Gamma$ if satisfiable in a $\delta$-model iff for a sufficiently large $\alpha$, the image $\Gamma[\delta, \alpha]$ is satisfiable in an $\mathrm{L}_{c p}$-model. A formula $\varphi$ is a consequence of $\Gamma$ over $\delta$-models iff $\varphi[\delta, \alpha]$ is a consequence of $\Gamma[\delta, \alpha]$ in $\mathrm{L}_{\mathrm{cp}}$. The ordinal $\alpha$ can be taken to be the supremum of the depths of formulas in $\Gamma, \varphi$.

\section{I EXAMPLES}

We will now return to the examples of flexible sets given in the introduction and show that they are indeed flexible. Recall that we use $\operatorname{Prop}(\Gamma)$ to denote the set of propositional variables that occur in some formula of $\Gamma$ outside a modal operator ${ }^{22}$ In each case, we must analyse the modality $\langle\Delta\rangle$ into three disjoint components $\Delta_{1}, \Delta_{2}$ and $\Delta_{3}$, ensuring that $\Delta_{1}$ and $\Delta_{3}$ contain no formulas with propositional variables in $\operatorname{Prop}(\Gamma)$. Furthermore, the description $\delta_{1}: \Delta \mapsto \Delta_{1}$

\footnotetext{
${ }^{22}$ The qualification 'outside a modal operator' was not needed when this was defined for $\mathrm{L}(\mathrm{C})$ formulas but in $\mathrm{L}_{\mathrm{cp}}$ the modal operators can also contain propositional variables and we need to exclude these from $\operatorname{Prop}(\Gamma)$.
} 
must be shown to be irreducible and $\delta_{2}: \Delta \mapsto \Delta_{2}$ must be shown to be finitary. We will call $\delta_{1}$ and $\delta_{2}$ the 'first component' and the 'second component', respectively ${ }^{23}$

I. $\Gamma$ contains only $\langle\Delta\rangle$ for finite $\Delta$. In this case, take $\Delta_{1}=\Delta_{3}=\emptyset$. Then $\Delta_{2}=\Delta$ is finite and so the second component is finitary. The modalities $\langle\Delta\rangle \varphi$ can then be eliminated, as shown above, with:

$$
\langle\Delta\rangle \varphi \leftrightarrow \bigvee_{\sigma \in \Sigma_{\Delta}}(\sigma \wedge\langle\emptyset\rangle(\sigma \wedge \varphi))
$$

with $\Sigma_{\Delta}$ the set of state descriptions of $\Delta$. This is the standard reduction of finitary ceteris paribus modalities to basic modal logic.

2. $\Gamma$ contains only one modality $\langle\Delta\rangle$ in which $\Delta=\operatorname{Prop} \backslash \operatorname{Prop}(\Gamma)$. In this case $\Delta=\Delta_{1} \cup \Delta_{2} \cup \Delta_{3}$ where $\Delta_{2}=\Delta_{3}=\emptyset . \Delta_{1}=\Delta$ is irreducible because it contains only propositional variables. The other condition for irreducibility is trivially satisfied because $\langle\Delta\rangle$ is the only modality occurring in $\Gamma$. Alternatively, if $\Delta$ is non-empty, we could take $\Delta_{1}=\{p\}$ for some $p \in \Delta$ and $\Delta_{3}$ to be the remaining propositional variables not in $\operatorname{Prop}(\Gamma)$. The propositional variable $p$ will serve as the sprinkle in Lemma I6.

3. $\Gamma$ contains many modalities $\left\langle\Delta_{i}\right\rangle$, with $\Delta_{0} \subset \Delta_{1} \subset \ldots \subseteq \operatorname{Prop} \backslash \operatorname{Prop}(\Gamma)$. In this case $\Delta_{i}=\Delta_{i 1} \cup \Delta_{i 2} \cup \Delta_{i 3}$ where $\Delta_{2}=\Delta_{3}=\emptyset$. The sets $\Delta_{i 1}=$ $\Delta_{i}$ are irreducible because they contain only propositional variables and for any non-empty set $\left\{\Delta_{j}\right\}_{j \in I}$ of these sets $\bigcup_{j \in J} \Delta_{j}=\Delta_{\max J}$ which is itself in the set. Alternatively, we could take $\Delta_{i 1}$ to $\left\{p_{i}\right\}$ where $p_{i}$ is any propositional variable occurring in $\Delta_{i}$ but not in $\Delta_{j}$ for $j<i$, and then put the remaining propositional variables in $\Delta_{i 3}$. Again, the variable $p_{i}$ is the sprinkle from Lemma I6

4. $\Gamma$ contains only the modalities $\langle\operatorname{Prop}\rangle$ and $\langle\emptyset\rangle$ and $\operatorname{Prop}(\Gamma)$ is finite. In this case, take Prop $=\operatorname{Prop}_{1} \cup \operatorname{Prop}_{2} \cup \operatorname{Prop}_{3}$ where $\operatorname{Prop}_{1}=\operatorname{Prop} \backslash \operatorname{Prop}(\Gamma)$, $\operatorname{Prop}_{2}=\operatorname{Prop}(\Gamma)$ and $\operatorname{Prop}_{3}=\emptyset$. Each component of $\emptyset$ is of course empty. Since Prop $_{2}$ is finite it is finitary for the same reason as was given in example I. Again, we could put only a sprinkle in Prop $_{1}$ and the rest in $\operatorname{Prop}_{3}$.

5. $\Gamma$ contains modalities $\langle\Delta\rangle$ in which $\Delta$ is any set of propositional variables, only a finite number of which are in $\operatorname{Prop}(\Gamma)$. This is only a slight generalisation of the last example: $\Delta_{1}=\Delta \backslash \operatorname{Prop}(\Gamma), \Delta_{2}=\Delta \cap \operatorname{Prop}(\Gamma)$ and $\Delta_{3}=\emptyset . \Delta_{2}$ is again finite and so the second component is finitary.

${ }^{23}$ As was previously noted, we do not need to pay special consideration to tracking in $\mathrm{L}_{\mathrm{cp}}$, because the $\leqslant$ order is given by $\subseteq$. 
6. $\Gamma$ contains a finite set of modalities $\left\langle\Delta_{1}\right\rangle, \ldots,\left\langle\Delta_{\mathfrak{n}}\right\rangle$ in which each $\Delta_{i}$ contains a cofinite set of propositional variables only finitely many of which are in $\operatorname{Prop}(\Gamma)$. The latter condition implies that $\operatorname{Prop}(\Gamma)$ is also finite. To say that $\Delta_{i}$ is cofinite is to say that Prop $\backslash \Delta_{i}$ is finite. Take $\Delta_{i 1}=\bigcap_{i=1}^{n} \Delta_{i} \backslash \operatorname{Prop}(\Gamma)$. This is the same set of propositional variables for each $i$, the cofinite set of those variables that occur in all of the $\Delta_{i}$ but not in $\operatorname{Prop}(\Gamma)$. Then take $\Delta_{i 2}=\Delta_{i} \backslash \Delta_{i 1}$ and $\Delta_{3}=\emptyset$. The first component is trivially irreducible - there is only one modal operator which contains only propositional variables - and the second component is finite and so finitary.

This example is a generalisation of the initial treatment of ceteris paribus preference logic by von Wright in [5]. In this system, a preference for, say, coffee $\mathrm{c}$ over tea $\mathrm{t}$ can be expressed as $\mathrm{cPt}$. According to von Wright, if surrounding conditions change in the world, then the preference might also change. I would change my preference for coffee over tea if the coffee you offered me was old and cold. But if the conditions stay constant, then I would always choose coffee over tea. To capture this idea, von Wright proposed that we should keep constant all the propositions that are not in the preference statement - constant in the sense that we have been investigating throughout this paper. In the case of $\mathrm{cPt}$, this means that we would keep constant all propositional variables other than $c$ and t. Let $\Delta=\operatorname{Prop} \backslash\{\mathrm{c}, \mathrm{t}\}$, then a ceteris paribus preference for coffee over tea can be expressed as $\mathrm{CP}_{\Delta} \mathrm{t}$. If Prop is infinite, than $\Delta$ is cofinite and von Wright's preference logic becomes a special case of the example under discussion.

7. $\{\varphi\}$ contains only modalities with cofinite or finite sets of propositional variables. The modalities with finite sets of propositional variables can be reduced to the modality $\langle\emptyset\rangle$ (which is a least element) and the modalities with cofinite sets can be reduced to a modality $\langle\Delta\rangle$ as in the previous example - with $\Delta$ the intersection of all sets occurring in modalities in $\varphi$ minus the propositional variables in $\varphi$ outside modalities. This leaves just two modalities $\langle\Delta\rangle$ and $\langle\emptyset\rangle$, neither of which contain propositional variables in $\operatorname{Prop}(\varphi)$.

The final examples gives a complete axiomatisation of a new fragment of $\mathrm{L}_{\mathrm{cp}}$ :

THEOREM 25 The fragment of $\mathrm{L}_{\mathrm{cp}}$ consisting of those formulas whose modal operators contain either a cofinite or finite set of propositional variables is completely (weakly) axiomatised by LCP.

None of these examples exploit the possibility of adding formulas in the $\Delta_{3}$ component. But further examples can be obtained by adding these at will, subject only to the tracking constraint. Moreover, the construction used in Lemma I5 enables us to generate examples with infinite $\Delta_{2}$. 


\section{CONCLUSION AND FURTHER WORK}

Given the lack of an axiomatisation for the standard logic of $\mathrm{L}_{\mathfrak{c p}}$, we have isolated (finitely characterisable) sufficient conditions for a set of formulas to be satisfiable. A set satisfying those conditions is called flexible and Theorem 22 shows that such a set is satisfiable whenever it is consistent. This motivated a new semantics for $L_{c p}$, under which the formulas $\Gamma$ in the modality $\langle\Gamma\rangle$ are taken to represent conditions on ceteris paribus equivalence that are necessary but not necessarily sufficient. Flexible Ceteris Paribus Logic was shown to have a strongly complete and natural axiomatisation.

The question of the weak axiomatisation of $\mathrm{L}_{c p}$ with standard semantics remains open but we conjecture that there is no such axiomatisation in the general case. When the sets $\Gamma$ in modalities $\langle\Gamma\rangle$ are required to be countable, there is more prospect of success, by analogy with the infinitary logic $L_{\omega_{1}, \omega}$, but we do not yet have a result.

Our approach to the initial problem involved the articulation of the more abstract language $\mathrm{L}(\mathcal{C})$ in which ceteris paribus conditions are represented only by parameters drawn from a partially ordered set $\mathcal{C}$. We think that this language is worthy of investigation in its own right. In particular, it would be interesting to impose more structure on $\mathcal{C}$, while falling short of the full expressive power of $\mathrm{L}_{c p}$. For example, it is fairly easy to see how the introduction of Boolean operators on parameters can be axiomatised, and the partial order on parameters could be used to represent a number of different relations (the expertise of agents, their influence in a community, etc.)

\section{REFERENCES}

[I] Patrick Blackburn, Johan F. A. K. van Benthem, and Frank Wolter. Handbook of Modal Logic, Volume 3 (Studies in Logic and Practical Reasoning). Elsevier Science Inc., New York, NY, USA, 2006.

[2] Patrick Blackburn, Maarten de Rijke, and Yde Venema. Modal Logic. Cambridge University Press, Cambridge, Mass., $200 \mathrm{I}$.

[3] Patrick Girard. Modal Logic for Belief and Preference Change. PhD thesis, Stanford University, 2008.

[4] Johan van Benthem, Patrick Girard, and Olivier Roy. Everything else being equal: a modal logic for ceteris paribus preferences. Fournal of philosophical logic, August 2008.

[5] G.H. von Wright. The Logic of Preference. Edinburgh, 1963. 
The Australasian fournal of Logic (ISSN I448-5052) disseminates articles that significantly advance the study of logic, in its mathematical, philosophical or computational guises. The scope of the journal includes all areas of logic, both pure and applied to topics in philosophy, mathematics, computation, linguistics and the other sciences.

Articles appearing in the journal have been carefully and critically refereed under the responsibility of members of the Editorial Board. Only papers judged to be both significant and excellent are accepted for publication.

The journal is freely available at the journal website at

http://www.philosophy.unimelb.edu.au/ajl/

All issues of the journal are archived electronically at the journal website.

SubSCRIPTIONS Individuals may subscribe to the journal by sending an email, including a full name, an institutional affiliation and an email address to the managing editor at ajl-editors@unimelb.edu.au Subscribers will receive email abstracts of accepted papers to an address of their choice. For institutional subscription, please email the managing editor at ajl-editors@unimelb.edu.au

Complete published papers may be downloaded at the journal's website at http: //www.philosophy.unimelb.edu.au/ajl/ The journal currently publishes in pdf format.

Submission The journal accepts submissions of papers electronically. To submit an article for publication, send the $\mathrm{IT}_{\mathrm{E}} \mathrm{X}$ source of a submission to a member of the editorial board. For a current list of the editorial board, consult the website.

The copyright of each article remains with the author or authors of that article. 